ESAIM: PROCEEDINGS, December 2008, Vol. 25, p. 44-67

E. Cancès, S. Faure, B. Graille, Editors

\title{
CONSTRUCTION AND ANALYSIS OF IMPROVED KIRCHOFF CONDITIONS FOR ACOUSTIC WAVE PROPAGATION IN A JUNCTION OF THIN SLOTS
}

\author{
PATRICK Joly ${ }^{1}$ AND Adrien SEMIN ${ }^{1,2}$
}

\begin{abstract}
In this paper, we analyze via the theory of matched asymptotics the propagation of a time harmonic acoustic wave in a junction of two thin slots. This allows us to propose improved Kirchoff conditions for the 1D limit problem. These conditions are analyzed and validated numerically.

Résumé. Dans cet article, nous utilisons la théorie des développements asymptotiques raccordés pour analyser la propagation d'ondes acoustiques à travers une jonction de deux fentes minces. Ceci nous permet de proposer des conditions de Kirchoff améliorées pour le problème limite 1D. Ces conditions sont analysées et validées numériquement.
\end{abstract}

\section{INTRODUCTION}

This article is intended to be the the first of a series of (at least) two papers on aymptotic models for time harmonic wave propagation in thin domains that are junctions of thin slots whose thickness $\varepsilon$ is small with respect to the wave length $\lambda$ and converges, when $\varepsilon$ tends to 0 , to a 1-dimensional graph. Intuitively, one expects that the solution of the original model converges (in a sense that will be made more precise later in this paper) to a 1D function defined on the limit graph. The homogeneous Neuman boundary condition is considered. The limit model is known for a long time : the limit solution satisfies the 1D time harmonic wave equation (namely the Helmholtz equation) and the so called Kirchoff conditions (in electricity) at each node of the graph : the solution is continuous at the node of the graph and the sum of fluxes at this node vanishes. Note however that the rigorous justification of such a result is quite recent : see the works of Rubinstein and Schatzman [8], [9] or Kuchment [5]. In these papers, eigenvalue problems are considered (but the results are quite similar to the ones that we shall investigate) : the convergence to the limit problem is established (without error estimate).

A natural question is to look for more accurate approximate models, i.e. models that would permit to identify not only the limit solution but also its first order (or higher order) expansion with respect to $\varepsilon$. As we shall see, this can be reduced to constructing improved Kirchoff conditions at the nodes ot the limit graph. The present work is a first contribution in this direction in the simplest possible case where we consider scalar wave propagation in a homogeneous medium composed by the junction of two thin 2D slots of the same thickness: in particular, the limit graph has only two branches and one node, From the technical point of view, the interest is that, even in this very simple case, the analysis is not so trivial and going beyond the limit problem requires some multi-scale asymptotic analysis in order to capture the non $1 \mathrm{D}$ phenomena that take place in the neighborhood of the junction. In this paper, in the spirit of the work of [3] for the junction between a thin slot and a half-space, we shall use the method of matched asymptotics (see also [1], [11] for more general references)

\footnotetext{
1 UMR POEMS, CNRS-ENSTA-INRIA, e-mail: patrick.joly@inria.fr \& adrien.semin@inria.fr

2 Equipe AN-EDP, Université Paris XI
} 
which is an interesting alternative to multiscale expansions (see [7] and [10] for the connection between the two approaches).

The outline of the article is as follows : in section 1, we present our model problem and state our main results, namely the improved Kirchoff conditions (17) and theorem 1.4. In section 2, we present the formal expansion of the solution using matched asymptotics (the techincal details are given in appendix A). The existence and uniqueness of the terms of the asymptotic expansion is established rigorously (the proof is detailed in appendix B), and error estimates are given. In section 3, we present the derivation of our improved conditions and show that they explain reflection phenomena (see also [6]) that are not visible with the limit model (this is illustrated with numerical results in section 3.3).

Above the intrinsic interest of the results, we also believe that this article has a pedagogical interest. Mathematical papers of matched asymptotics or multi-scale expansions are often very hard to read, in particular because of the needed notations and the high technicality of the mathematical developments. The simplicity of the model problem we consider here is very useful to simplify these aspects, which allowed us to make a presentation that we hope to be digest, without sacrifying the mathematical rigor. In order to avoid writing a much longer paper, we have chosen to avoid the detailed presentation of some of the analytical developments of our work. The reader will find these details in the technical report [2] that is a kind of expanded version of the present paper.

In a forthcoming paper, we shall look at the case of a general limit graph in the even more interesting case of the time dependent problem.

\section{Model PROBlem AND MAIN RESUlts}

\subsection{The model problem}

In this section we introduce the geometry and the equations of our problem. We consider a domain made of the junction of two slots (see figure 1). More precicely, we consider the union of two thin rectangles with respective lengths $L_{-}$and $L_{+}$and thickness $\varepsilon$, the small parameter in the analysis, with a junction zone. A geometrical characteristic of this domain is the angle $2 \alpha$ between the two thin slots. For the analysis, we consider in fact a family of such thin domains denoted $\Omega^{\varepsilon}$ with varying $\varepsilon$. We make the choice (this has an influence on the asymptotic analysis) that one part of the boundary of $\Omega^{\varepsilon}$ remains fixed, namely the two segments $S^{-}$and $S^{+}$ that intersect at the reentrant corner of the junction zone. Analytically, we have:

$$
\Omega^{\varepsilon}=\Omega_{-}^{\varepsilon} \cup \Omega_{+}^{\varepsilon} \cup J_{\alpha}^{\varepsilon} \quad 1
$$

with $J_{\alpha}^{\varepsilon}=\varepsilon \widehat{J}_{\alpha}$ where $\widehat{J}_{\alpha}$ is the normalized junction presented in figure 2 and

$$
\left\{\begin{array}{l}
\Omega_{ \pm}^{\varepsilon}=\left\{\mathbf{x}=(x, y) \in \mathbb{R}^{2} / 0< \pm \mathbf{x} \cdot \mathbf{t}^{ \pm}<L_{ \pm},-\varepsilon<\mathbf{x} \cdot \mathbf{n}^{ \pm}<0\right\} \\
\mathbf{t}^{-}=(1,0)^{t}, \quad \mathbf{n}^{-}=(0,1)^{t}, \quad \mathbf{t}^{+}=(\cos (2 \alpha), \sin (2 \alpha))^{t}, \quad \mathbf{n}^{+}=(-\sin (2 \alpha), \cos (2 \alpha))^{t},
\end{array}\right.
$$

The problem we consider is : find $u^{\varepsilon} \in \mathrm{H}^{1}\left(\Omega^{\varepsilon} ; \mathbb{C}\right)$ such as

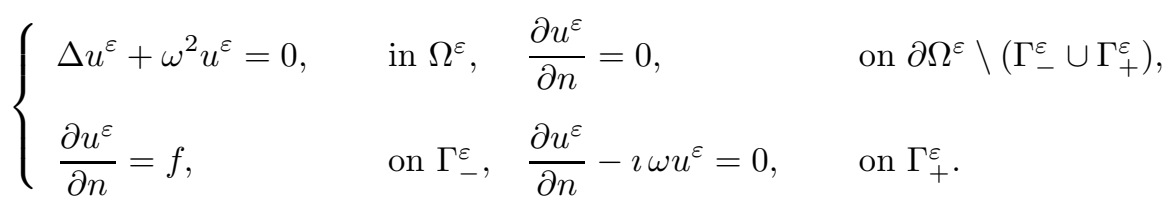

\footnotetext{
${ }^{1}$ To be more precise, the closure of $\Omega^{\varepsilon}$ is equal to the union of the closure of the other sets
} 


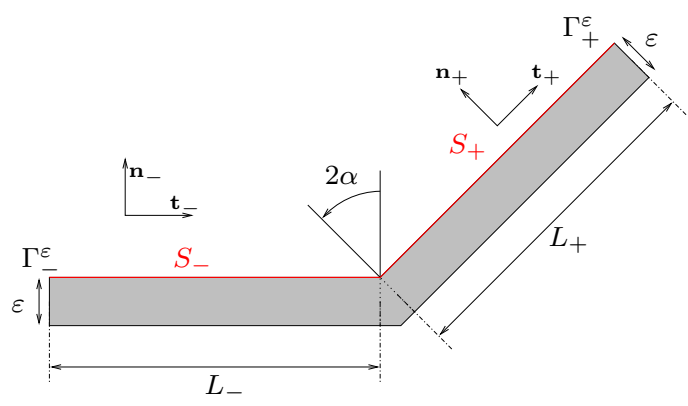

Figure 1. Configuration of the domain

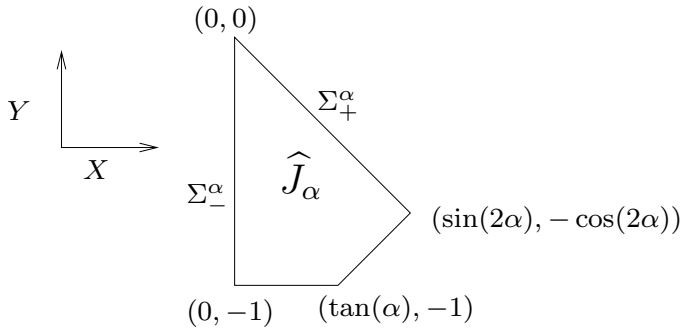

Figure 2. The junction $\widehat{J}_{\alpha}$

where the source term $f$ is a given constant (i.e. a constant function along $\Gamma_{-}^{\varepsilon}$ ) and $n$ denotes the outward unit vector along $\partial \Omega^{\varepsilon}$.

Remark 1.1. The boundary condition on $\Gamma_{+}^{\varepsilon}$ can be interpreted as an approximate radiation condition (see for instance [4] for more details).

\subsection{The 1D limit problem}

When $\varepsilon$ tends to 0 , the domain $\Omega^{\varepsilon}$ "degenerates" into a " $1 \mathrm{D}$ domain" namely the union of the two segments $S_{-}$and $S^{+}$(see figure 1). Intuitively, one expects that the solution $u^{\varepsilon}$ "converges" to a "1D function", namely a function of the arclength $s$ along $S_{-} \cup S_{+}$, solution of a "1D problem". It remains to give a more precise mathematical meaning to such a statement. To describe the "limit problem" inside the slots, we will use local normalized (tangential and normal) coordinates $(s, \widehat{\nu})$, that express that $\Omega_{+}^{\varepsilon}$ and $\Omega_{-}^{\varepsilon}$ are isomorphic to the rectangles

through the maps

$$
\left.\widehat{\Omega}_{+}=\right] 0, L_{+}[\times]-1,0\left[, \quad \widehat{\Omega}_{-}=\right]-L_{-}, 0[\times]-1,0[,
$$

$$
\mathbf{x}=(x, y) \mapsto(s, \widehat{\nu})=\left(\mathbf{x} \cdot \mathbf{t}^{ \pm}, \mathbf{x} \cdot \mathbf{n}^{ \pm} / \varepsilon\right) \quad \text { from } \Omega_{ \pm}^{\varepsilon} \text { into } \widehat{\Omega}_{ \pm}
$$

Let us introduce $\left.u^{0}(s):\right]-L_{-}, L_{+}[\rightarrow \mathbb{C}$ be the solution of the 1D Hemholtz equation (our 1D limit problem):

$$
\begin{cases}\frac{\partial^{2} u^{0}}{\partial s^{2}}+\omega^{2} u^{0}=0, & \text { in }]-L_{-}, L_{+}[, \\ -\frac{\partial u^{0}}{\partial s}\left(-L_{-}\right)=f, & {\left[\frac{\partial u^{0}}{\partial s}-\imath \omega u^{0}\right]\left(L_{+}\right)=0,}\end{cases}
$$

from which we define the $2 \mathrm{D}$ functions $\widehat{u}_{ \pm}^{0}: \widehat{\Omega}_{ \pm} \rightarrow \mathbb{C}$ such that

$$
\widehat{u}_{ \pm}^{0}(s, \widehat{\nu})=u^{0}(s) \quad \text { in } \widehat{\Omega}_{ \pm}
$$

Next, we introduce the "rescaled" exact solutions in $\widehat{\Omega}_{ \pm}$

$$
\widehat{u}_{ \pm}^{\varepsilon}(s, \widehat{\nu})=u^{\varepsilon}(x, y) \quad \text { for }(s, \widehat{\nu})=\left(\mathbf{x} \cdot \mathbf{t}^{ \pm}, \mathbf{x} \cdot \mathbf{n}^{ \pm} / \varepsilon\right)
$$

as well as in the normalized junction

$$
\widehat{U}^{\varepsilon}(\widehat{\mathbf{x}})=u^{\varepsilon}(\varepsilon \widehat{\mathbf{x}}) \quad \text { in } \widehat{J}_{\alpha}
$$

The precise meaning of the convergence of $u^{\varepsilon}$ to $u^{0}$ is given in the following theorem : 
Theorem 1.2. The functions $\widehat{u}_{ \pm}^{\varepsilon}$ converge to the "1D functions" $\widehat{u}_{ \pm}^{0}$ in $\mathrm{H}^{1}\left(\widehat{\Omega}_{ \pm}\right)$and the function $\widehat{U}^{\varepsilon}$ converges to the constant $\widehat{u}^{0}(0)$ in $\mathrm{H}^{1}\left(\widehat{J}_{\alpha}\right)$. Moreover, there exists a positive constant $C$, independent of $\varepsilon$ such that:

$$
\sum_{ \pm}\left\|\widehat{u}_{ \pm}^{\varepsilon}-\widehat{u}_{ \pm}^{0}\right\|_{\mathrm{H}^{1}\left(\widehat{\Omega}_{ \pm}\right)}+\left\|\widehat{U}^{\varepsilon}-\widehat{u}^{0}(0)\right\|_{\mathrm{H}^{1}\left(\widehat{J}_{\alpha}\right)} \leqslant C \varepsilon
$$

The proof of the convergence result of theorem 1.2 is relatively elementary (see [2]) and will not be reproduced here. It consists essentially in deriving uniform bounds (a priori estimates) for the exact solutions, extracting converging subsequences by compactness, and identifying the limit solution thanks to appropriate test functions (typically '1D test functions' functions in the slots). The proof of the error estimate is much less trivial and will be in fact a consequence of the asymptotic analysis developed in the present work. Let us remark that this estimate can not be deduced from the results of [5] or [8].

A first immediate (and a priori surprising) observation is that the limit solution $u^{0}$ does not see the geometry of the junction: in particular, it is independent of the angle $\alpha$ ! Physically it means that, for the limit problem, the incident wave emitted at $s=-L^{-}$by the source term $f$ does not produce any reflection when it reaches the junction at $s=0:$ it is completely transmitted. For $\varepsilon>0$, it is clear that there exists a reflected wave whose amplitude is expected to be an increasing function of $\alpha$. This raises the following question: does there exist an "improved 1D model" whose solution would provide a better approximation to the exact solution than $u^{0}$, and would in particular permit us to observe a reflected wave ? The answer to this question (at least one possible answer) is the object of the following subsection.

To better understand how the 1D problem will be modified, one has to interpret the limit solution $u^{0}$ as the solution of a transmission problem between two 1D Helmholtz equations in the segments $]-L^{-}, 0[$ and $] 0, L^{+}[$ with the help of the transmission conditions:

$$
\left\{\begin{array}{l}
{\left[u^{0}\right]:=u^{0}\left(0^{+}\right)-u^{0}\left(0^{-}\right)=0,} \\
{\left[\frac{\partial u^{0}}{\partial s}\right]:=\frac{\partial u^{0}}{\partial s}\left(0^{+}\right)-\frac{\partial u^{0}}{\partial s}\left(0^{-}\right)=0 .}
\end{array}\right.
$$

These are nothing but the particular version of the well-known "Kirchoff conditions" at a node where only two branches of a graph is connected. Thus, our question could be rephrased as follows : does there exist "improved Kirchoff conditions" that would lead to a better approximation of the true solution than $u^{0}$ ?

\subsection{An improved 1D approximate model}

To describe our improved model, we need to introduce some additional notation. The construction of the improved solution will use the solution of an auxiliary (frequency independent) boundary value problem posed in the normalized junction $\widehat{J}_{\alpha}$, namely the problem which consists in finding $\mathcal{W}_{\alpha} \in \mathrm{H}^{1}\left(\widehat{J}_{\alpha}\right)$, with mean-value 0

$$
\int_{\widehat{J}_{\alpha}} \mathcal{W}_{\alpha}=0
$$

that solves the boundary value problem (note that $\mathcal{W}_{\alpha}$ depends on $\alpha$ through $\widehat{J}_{\alpha}$ )

$$
\begin{cases}\Delta \mathcal{W}_{\alpha}=0, & \text { in } \widehat{J}_{\alpha}, \quad \frac{\partial \mathcal{W}_{\alpha}}{\partial n}=0, \\ \frac{\partial \mathcal{W}_{\alpha}}{\partial n}+T_{ \pm} \mathcal{W}_{\alpha}= \pm 1, & \text { on } \Sigma_{ \pm}^{\alpha}\end{cases}
$$


where, once $\Sigma_{ \pm}^{\alpha}$ has been identified to the segment $]-1,0\left[, T_{ \pm}\right.$is nothing but the nonlocal operator defined as

$$
\mid \begin{array}{llll}
T: & \mathrm{H}^{\frac{1}{2}}(]-1,0[) & \rightarrow & \mathrm{H}^{-\frac{1}{2}}(]-1,0[) \\
& \varphi=\sum_{p=0}^{\infty} \varphi_{p} w_{p} & \mapsto T \varphi=\sum_{p=0}^{\infty} \pi p \varphi_{p} w_{p}
\end{array}
$$

where $w_{p}$ is the orthonormal basis of $\mathrm{L}^{2}(]-1,0[)$ given by

$$
w_{0}(\widehat{\nu})=1, \quad w_{p}(\widehat{\nu})=\sqrt{2} \cos p \pi \widehat{\nu}, \quad p=1,2,3, \cdots
$$

It is easy to see, with Lax-Milgram's lemma, that $(11,10)$ determines uniquely $\mathcal{W}_{\alpha}$ (see [2]). This function will appear in the approximate problem through the constant (which is uniquely determined even without (10)):

$$
K(\alpha)=\int_{\Sigma_{+}^{\alpha}} \mathcal{W}_{\alpha}-\int_{\Sigma_{-}^{\alpha}} \mathcal{W}_{\alpha}
$$

It seems difficult to get a closed formula for $K(\alpha)$. However, we have been able to prove the following lemma (that we shall use in section 3.2):

Lemma 1.3. The function $\alpha \in] 0, \frac{\pi}{2}\left[\mapsto \frac{K(\alpha)}{\tan \alpha}\right.$ is smooth, strictly decreasing and satisfies

$$
\lim _{\alpha \rightarrow 0} \frac{K(\alpha)}{\tan \alpha}=1, \quad \lim _{\alpha \rightarrow \frac{\pi}{2}} \frac{K(\alpha)}{\tan \alpha}=0 .
$$

Proof. The result is obtained by studying more carefully the boundary value problem $(11,10)$ when $\alpha$ varies, via variational techniques. We refer the reader to [2].

The new 1D approximate problem that we consider consists in finding $\left.\tilde{u}^{\varepsilon}:\right]-L^{-}, L^{+}[\rightarrow \mathbb{C}$, solution of the 1D Helmholtz equation in each segment $]-L^{-}, 0[$ and $] 0, L^{+}[$(note the difference with (4-(i)) ):

$$
\left.\frac{\partial^{2} \tilde{u}^{\varepsilon}}{\partial s^{2}}+\omega^{2} \tilde{u}^{\varepsilon}=0, \quad \text { in }\right]-L^{-}, 0[\cup] 0, L^{+}[,
$$

satisfies the same boundary conditions than $u^{0}$ at $s= \pm L^{ \pm}$, i.e. (4-(ii)), and finally the transmission conditions

$$
\begin{cases}{\left[\tilde{u}^{\varepsilon}\right]=\varepsilon K(\alpha)\left\langle\frac{\partial \tilde{u}^{\varepsilon}}{\partial s}\right\rangle,} & \text { where }\left\langle\frac{\partial \tilde{u}^{\varepsilon}}{\partial s}\right\rangle:=\frac{1}{2}\left(\frac{\partial \tilde{u}^{\varepsilon}}{\partial s}\left(0^{+}\right)+\frac{\partial \tilde{u}^{\varepsilon}}{\partial s}\left(0^{-}\right)\right), \\ {\left[\frac{\partial \tilde{u}^{\varepsilon}}{\partial s}\right]=-\varepsilon \omega^{2} \tan \alpha\left\langle\tilde{u}^{\varepsilon}\right\rangle,} & \text { where }\left\langle\tilde{u}^{\varepsilon}\right\rangle:=\frac{1}{2}\left(\tilde{u}^{\varepsilon}\left(0^{+}\right)+\tilde{u}^{\varepsilon}\left(0^{-}\right)\right) .\end{cases}
$$

The conditions (17) are clearly first order modifications of the transmission conditions (9). To understand in which sense they provide a better approximation than (9), following the definition of $\widehat{u}^{0}$ from $u^{0}$, we reconstruct from $\tilde{u}^{\varepsilon}$ a $2 \mathrm{D}$ solution in the normalized slots as

$$
\widehat{u}_{ \pm}^{\varepsilon, a p p}(s, \widehat{\nu})=\tilde{u}^{\varepsilon}(s) \quad \text { in } \widehat{\Omega}_{ \pm}
$$

and, for $0<\delta<\delta^{*}:=\max \left\{L_{+}, L_{-}\right\}$, we define

$$
\left.\widehat{\Omega}_{+}^{\delta}=\right] \delta, L_{+}[\times]-1,0\left[, \quad \widehat{\Omega}_{-}^{\delta}=\right]-L_{-},-\delta[\times]-1,0[.
$$


Theorem 1.4. For any $\varepsilon>0$, the boundary value problem $(16,4-($ ii $), 17)$ is well posed in $\mathrm{H}^{1}(]-L^{-}, 0[\cup] 0, L^{+}[)$. Moreover, for any $0<\delta<\delta^{*}$, there exists a constant $C_{\delta}$, independent of $\varepsilon$ such that

$$
\sum_{ \pm}\left\|\widehat{u}_{ \pm}^{\varepsilon, a p p}-\widehat{u}_{ \pm}^{\varepsilon}\right\|_{\mathrm{H}^{1}\left(\widehat{\Omega}_{ \pm}^{\delta}\right)} \leqslant C_{\delta} \varepsilon^{3}
$$

Remark 1.5. Contrary to what happens in theorem 1.2 , it is not possible to take $\delta=0$ in (20) (in other words the constant $C_{\delta}$ blows up when $\delta$ tends to 0$)$. This is due to the apparition of some boundary layer in the neighborhood of the junction (see [2] for more details).

\section{The FORMAL EXPANSIONS}

As we said in the introduction, as the problem is multi-scale, it is not possible to write a uniform expansion for the solution everywhere in the domain $\Omega^{\varepsilon}$. The method of matched asymptotics will lead us, we have to consider three distinct zones, respectively two slot zones and a junction zone, in which different expansions will be obtained. However, contrarily to the naive intuition, this domain decomposition does not correspond to the partition (1) of $\Omega^{\varepsilon}$ : in the method of matched asymptotics, the different domains must overlap, the idea being that the different expansions must "coincide" in the overlaping zones.

\subsection{An overlaping domain decomposition}

In the following, we will denote by $\mathcal{C}$ the class of positive continuous functions of $\varepsilon>0$ that tend to 0 when $\varepsilon \rightarrow 0$, less rapidly than $\varepsilon|\ln (\varepsilon)|$ (A typical example is $\varepsilon^{\beta}$, with $\beta$ strictly less than 1 ).

$$
\mathcal{C}=\left\{\varphi: \mathbb{R}_{+}^{*} \rightarrow \mathbb{R}_{+}^{*} / \lim _{\varepsilon \rightarrow 0} \varphi(\varepsilon)=0 \text { and } \lim _{\varepsilon \rightarrow 0} \varphi(\varepsilon) /(\varepsilon|\ln (\varepsilon)|)=+\infty\right\}
$$

Given $\varphi$ in $\mathcal{C}$, we define

$$
\Omega_{ \pm}(\varepsilon)=\left\{\mathbf{x}=(x, y) \in \Omega^{\varepsilon} / \varphi(\varepsilon)< \pm \mathbf{x} \cdot \mathbf{t}^{ \pm}<L_{ \pm}\right\} \quad\left(\subset \Omega_{ \pm}^{\varepsilon}\right)
$$

The junction zone is defined by

$$
J_{\alpha}(\varepsilon)=\varepsilon \widehat{J}_{\alpha} \cup \bigcup_{ \pm}\left\{(x, y) \in \Omega^{\varepsilon} \text { such that } 0 \leqslant \pm \mathbf{x} \cdot \mathbf{t}^{ \pm}<2 \varphi(\varepsilon)\right\}, \quad\left(\supset J_{\alpha}^{\varepsilon}\right)
$$

in such a way that we have $\Omega^{\varepsilon}=\Omega_{+}(\varepsilon) \cup \Omega_{-}(\varepsilon) \cup J(\varepsilon)$ with two overlaping regions (see the figure 2.1)

$$
\mathcal{O}_{ \pm}(\varepsilon):=\left\{(x, y) \in \Omega^{\varepsilon} \text { such that } \varphi(\varepsilon)< \pm \mathbf{x} \cdot \mathbf{t}^{ \pm}<2 \varphi(\varepsilon)\right\}
$$

The mapping

$$
\mathbf{x}=(x, y) \mapsto(s, \widehat{\nu})=\left(\mathbf{x} \cdot \mathbf{t}^{ \pm}, \mathbf{x} \cdot \mathbf{n}^{ \pm} / \varepsilon\right)
$$

maps the domain $\Omega_{ \pm}(\varepsilon)$ into the rectangle $\widehat{\Omega}_{ \pm}(\varepsilon)$ with

$$
\left.\widehat{\Omega}_{+}(\varepsilon)=\right] \varphi(\varepsilon), L^{+}[\times]-1,0\left[, \quad \widehat{\Omega}_{-}(\varepsilon)=\right]-L^{-},-\varphi(\varepsilon)[\times]-1,0[,
$$

Note that $\widehat{\Omega}_{ \pm}(\varepsilon)$ increases when $\varepsilon$ decreases and converges to $\widehat{\Omega}_{ \pm}$when $\varepsilon$ tends to 0 . In the same way,

$$
\mathbf{x}=(x, y) \mapsto \widehat{\mathbf{x}}=\mathbf{x} / \varepsilon
$$


maps $J_{\alpha}(\varepsilon)$ onto $\widehat{J}_{\alpha}(\varepsilon)$ (see the figure 3 ), a domain which increases when $\varepsilon$ decreases and converges to the unbounded domain:

$$
\widehat{J}_{\alpha, \infty}=\widehat{J}_{\alpha} \cup \widehat{B}^{+} \cup \widehat{B}^{-}, \quad \widehat{B}^{ \pm}=\left\{\widehat{\mathbf{x}} / \pm \widehat{\mathbf{x}} \cdot \mathbf{t}^{ \pm} \geqslant 0, \widehat{\mathbf{x}} \cdot \mathbf{n}^{ \pm} \in\right]-1,0[\}
$$

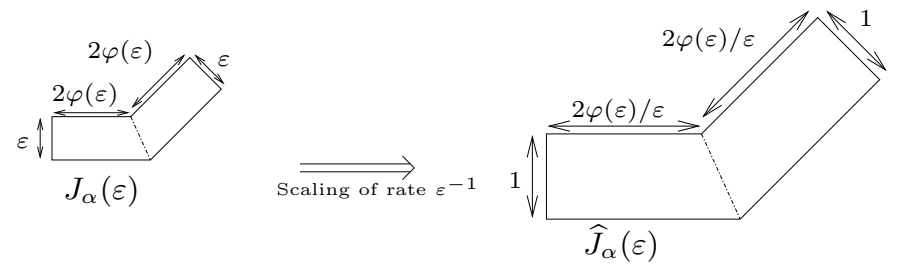

Figure 3. The near-field zone

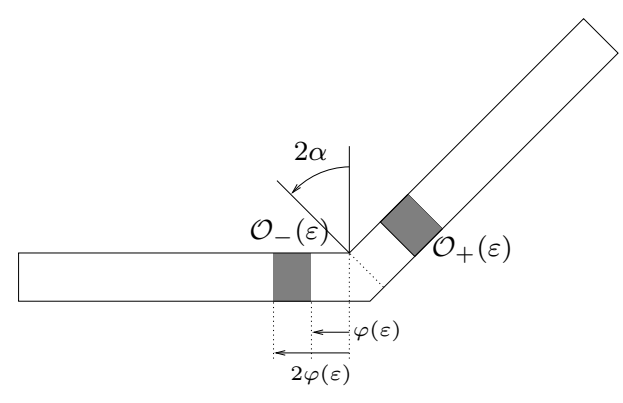

Figure 4. The overlaping zones $\mathcal{O}_{ \pm}(\varepsilon)$

\subsection{Local expansions and basic equations}

We formulate our ansatz for the asymptotic expansions which consists, in each zone after scaling ((25) or (26)), in looking for power series expansions with respect to $\varepsilon \omega$. In other words, we look for functions

$$
u_{ \pm}^{k}: \widehat{\Omega}_{ \pm} \rightarrow \mathbb{C} \quad \text { and } \quad U^{k}: \widehat{J}_{\alpha, \infty} \rightarrow \mathbb{C}, \quad k \in \mathbb{N},
$$

such that, at least formally

$$
\begin{aligned}
& u^{\varepsilon}(s, \varepsilon \widehat{\nu})=\sum_{k=0}^{\infty}(\varepsilon \omega)^{k} u_{ \pm}^{k}(s, \widehat{\nu})+o(\varepsilon \omega)^{\infty}, \quad \text { in } \widehat{\Omega}_{ \pm}(\varepsilon), \\
& u^{\varepsilon}(\varepsilon \widehat{\mathbf{x}})=\sum_{k=0}^{\infty}(\varepsilon \omega)^{k} U^{k}(\widehat{\mathbf{x}})+o(\varepsilon \omega)^{\infty}, \quad \text { in } \widehat{J}_{\alpha}(\varepsilon) .
\end{aligned}
$$

It remains to obtain the equations that will determine the functions $u_{ \pm}^{k}$ and $U^{k}$. For the $u_{ \pm}^{k}$ 's, we substitute formally the expansion (28) in the 2D Helmholtz equation written in $\Omega_{ \pm}(\varepsilon)$, using the scaled coordinates $(s, \widehat{\nu})$, and we identify the terms with the same power of $\varepsilon$. Straightforward manipulations lead to :

$$
\frac{\partial^{2} u_{ \pm}^{0}}{\partial \widehat{\nu}^{2}}=0, \quad \frac{\partial^{2} u_{ \pm}^{1}}{\partial \widehat{\nu}^{2}}=0, \quad \frac{\partial^{2} u_{ \pm}^{k}}{\partial s^{2}}+\omega^{2} u_{ \pm}^{k}+\frac{\partial^{2} u_{ \pm}^{k+2}}{\partial \widehat{\nu}^{2}}=0, \quad k \geqslant 0 .
$$

while the Neumann boundary condition along the "lateral" sides of lead to :

$$
\frac{\partial u_{ \pm}^{k}}{\partial \widehat{\nu}}(\widehat{s},-1)=\frac{\partial u_{ \pm}^{k}}{\partial \widehat{\nu}}(\widehat{s}, 0)=0, \quad \pm \widehat{s}>0, \quad k \geqslant 0 .
$$

From (30) and (31), we deduce that

$$
\text { (i) } \quad u_{ \pm}^{k}(s, \widehat{\nu})=u_{ \pm}^{k}(s), \quad \text { (ii) } \quad \frac{\partial^{2} u_{ \pm}^{k}}{\partial s^{2}}+\omega^{2} u_{ \pm}^{k}=0, \quad \pm s \in\left[0, L^{ \pm}[, \quad k \geqslant 0 .\right.
$$

These properties are easily established by induction on $k$. Indeed, for $k=0,1$, the first two equations of (30) combined with (31) show that $u_{ \pm}^{0}$ and $u_{ \pm}^{1}$ are independent of $\widehat{\nu}$. Then, integrating the third equation of (30) 
written for $k=0$ (respectively $k=1$ ) with respect to $\widehat{\nu}$ (from -1 to 0 ) and using the boundary conditions (31) for $k=2$ and 3 , we see that $u_{ \pm}^{0}$ (respectively $\left.u_{ \pm}^{1}\right)$ satisfies $(32-(i i)$ ).

Assume that (32) holds up tp $k=p$. Then, the third equations of (30) written for $k=p-1$ combined with (31) written for $k=p+1$ show that $u_{ \pm}^{p+1}$ is independent of $\widehat{\nu}$. Next, integrating the third equation of (30) written for $k=p+1$ with respect to $\widehat{\nu}$ and using the boundary conditions (31) for $k=p+3$ leads to (32-(ii)) for $k=p+1$.

Moreover, from the boundary conditions on $\Gamma_{ \pm}$in $(3)$, we deduce

$$
\text { (i) }\left(\frac{\partial u_{+}^{k}}{\partial s}-\imath \omega u_{+}^{k}\right)\left(L_{+}\right)=0, \quad k \geqslant 0, \quad(i i) \quad \frac{\partial u_{-}^{k}}{\partial s}\left(-L_{-}\right)=-f \quad \text { for } k=0, \quad=0 \quad \text { for } k \geqslant 1 .
$$

To obtain the equations for the $U^{k}$ 's, we substitute formally the expansion (29) in the 2D Helmholtz equation written in $J(\varepsilon)$, using the scaled coordinates $\widehat{x}$, and we identify the terms with the same power of $\varepsilon$. This permits to see that the $U^{k}$ 's satisfy embedded Laplace's equations

$$
\Delta U^{0}=0, \quad \Delta U^{1}=0, \quad \Delta U^{k}+U^{k-2}=0, \quad k \geqslant 2, \quad \text { in } \widehat{J}_{\alpha, \infty} .
$$

with Neumann boundary conditions

$$
\frac{\partial U^{k}}{\partial n}=0, \quad \text { on } \partial \widehat{J}_{\alpha, \infty}, \quad k \in \mathbb{N}
$$

Remark 2.1. In the sequel, we shall adopt the convention that all quantities super-indexed by $k$ (such as $U^{k}$, $\left.u_{ \pm}^{k}, \ldots\right)$ are 0 for negative values of $k$. This will be useful to simplify some formulas. For instance, with this convention the last equation of (34) is also valid for $k=0,1$.

\subsection{Matching conditions}

Equations $(32)$ to $(35)$ are not sufficient to characterize the functions $\left(u_{ \pm}^{k}, U^{k}\right)$ 's: we miss boundary conditions at $s=0$ for the $u_{ \pm}^{k}$ 's and additionnal conditions for the $U^{k}$ 's. These conditions, namely the matching conditions, will couple the $u_{ \pm}^{k}$ 's and the $U^{k}$ 's. To derive them, one writes that the two expansions (28) and (29) must coincide in the overlaping zones $(24)$, i. e. denoting $U_{ \pm}^{k}$ the restrictions of $U^{k}$ to the two semi-strips $\widehat{B}_{ \pm}$(see figure 5) :

$$
\sum_{k=0}^{\infty}(\varepsilon \omega)^{k} U_{ \pm}^{k}(s / \varepsilon, \widehat{\nu} / \varepsilon)+o(\varepsilon \omega)^{\infty}=\sum_{k=0}^{\infty}(\varepsilon \omega)^{k} u_{ \pm}^{k}(s, \widehat{\nu} / \varepsilon)+o(\varepsilon \omega)^{\infty} \quad \text { in } \mathcal{O}^{ \pm}(\varepsilon)
$$

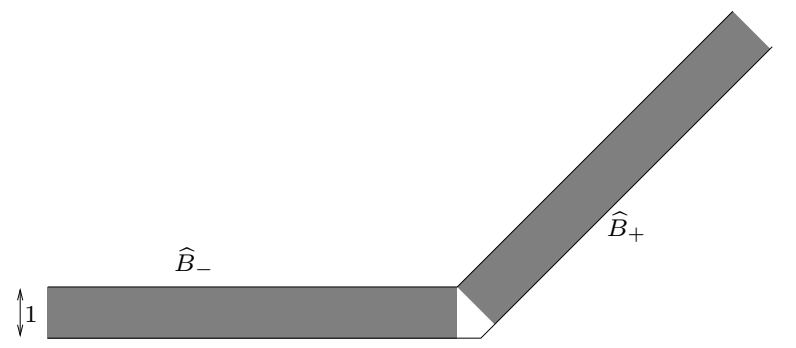

Figure 5. The subdomains $\widehat{B}_{ \pm}$ 
The calculations that are detailed in appendix A (which constitutes the body of the matched asymptotics procedure), leads to the following conditions :

$$
\begin{aligned}
& U^{k} \text { grows as most polynomially at infinity in } \widehat{J}_{\alpha, \infty} \\
& \left\{\begin{array}{lll}
(\mathcal{D}) & u_{ \pm}^{k}(0)=\int_{\Sigma_{ \pm}^{\alpha}} U^{k}, & k \geqslant 0, \\
(\mathcal{N}) & \frac{\partial u_{ \pm}^{k-1}}{\partial s}(0)= \pm \omega \int_{\Sigma_{ \pm}^{\alpha}} \frac{\partial U^{k}}{\partial \mathbf{n}}, & k \geqslant 0 .
\end{array}\right.
\end{aligned}
$$

where $\mathbf{n}$ denotes the exterior normal vector to $\widehat{J}_{\alpha}$.

\subsection{Existence and uniqueness of the formal expansion}

The next result express that the matching conditions (38) and the growth conditions (37) are sufficient to define entirely the terms of the asymptotic expansions (28) and (29). The precise result is the following :

Theorem 2.2. There exists a unique family

$$
\left\{\left(u_{+}^{k}, u_{-}^{k}, U^{k}\right) \in \mathrm{H}^{1}\left(\widehat{\Omega}_{+}\right) \times \mathrm{H}^{1}\left(\widehat{\Omega}_{-}\right) \times \mathrm{H}_{l o c}^{1}\left(\widehat{J}_{\alpha, \infty}\right), \quad k \geqslant 0\right\}
$$

satisfying (32, 33, 34, 35), the matching conditions (38) and the growth conditions (37).

Due to its technical nature, the proof of this result is given in the appendix B.

\subsection{Error estimates for the truncated expansion}

The 1D function $\quad u^{\varepsilon, k}(s)=\sum_{m=o}^{k}(\varepsilon \omega)^{k} u^{k}(s), \quad-L_{-} \leqslant s \leqslant L_{+}, \quad$ with $u^{k}(s):=u_{ \pm}^{k}(s) \quad$ if $\left.\pm s \in\right] 0, L_{ \pm}[$,

is a natural good candidate to provide an accurate $1 \mathrm{D}$ approximation of the exact solution. The precise meaning of this can be expressed in terms of an error estimate which generalizes the estimate in the case $\mathrm{k}=0$.

From $u^{\varepsilon, k}$ we define the 2D functions $\widehat{u}^{\varepsilon, k}$ in the normalized slots $\widehat{\Omega}_{ \pm}$

$$
\widehat{u}_{ \pm}^{\varepsilon, k}(s, \widehat{\nu})=u^{\varepsilon, k}(s) \quad \text { in } \widehat{\Omega}_{ \pm}
$$

that we compare to the "rescaled" exact solutions $\widehat{u}_{ \pm}^{\varepsilon}$ (see (6)) in the domains $\widehat{\Omega}_{ \pm}^{\delta}$ (see (19)).

Theorem 2.3. For any $0<\delta<\delta^{*}$ and any $k \geqslant 1$, there exists a constant $C_{\delta}^{k}>0$, independent of $\varepsilon$ such that:

$$
\sum_{ \pm}\left\|\widehat{u}_{ \pm}^{\varepsilon}-\widehat{u}_{ \pm}^{k, \varepsilon}\right\|_{H^{1}\left(\widehat{\Omega}_{ \pm}^{\delta}\right)} \leqslant C_{\delta}^{k} \varepsilon^{k+1}
$$

Proof. We only sketch the proof (which is not the main purpose of this paper) and refer the reader to [2] for the technical details. The ideas of the proof are similar to the arguments of [3]. The estimate (41) is a local error estimate in the slots. It can be deduced from a "global" error estimate that is obtained as follows:

- We consider $\varphi \in \mathcal{C}$ (going back to section 2.1, we consider $\varphi_{ \pm}=\varphi$ ) and introduce a 1D cut-off function

$$
\left.\Phi_{\varepsilon}(s)=\Phi\left(\frac{s}{\varphi(\varepsilon)}\right) \quad \text { with } \quad \Phi \in \mathcal{C}_{0}^{\infty}(\mathbb{R}), \quad \operatorname{supp} \Phi \in\right]-2,2[, \quad \Phi(s)=1 \text { in }[-1,1]
$$


from which we construct a $2 \mathrm{D}$ cut-off function $\widehat{\Phi}_{\varepsilon}$ (in an obvious way) in the domain

$$
\widehat{\Omega}_{\alpha}=\widehat{\Omega}_{+} \cup \widehat{\Omega}_{-} \cup \widehat{J}_{\alpha}
$$

- Next we propose the global approximation in

$$
\widehat{u}_{a p p}^{\varepsilon, k}=\left(1-\widehat{\Phi}_{\varepsilon}\right) \widehat{u}_{ \pm}^{\varepsilon, k}+\widehat{\Phi}_{\varepsilon} \widehat{U}^{\varepsilon, k} \quad \text { where } \quad \widehat{U}^{\varepsilon, k}:=\sum_{m=o}^{k}(\varepsilon \omega)^{m} U^{m} \quad \text { in } \widehat{J}_{\alpha, \infty}
$$

If we denote $\widehat{u}^{\varepsilon}$ the "rescaled" exact solution (6) in $\widehat{\Omega}$, namely the function that coincides with $\widehat{u}^{\varepsilon}$ $(\operatorname{cf}(6))$ in $\widehat{\Omega}_{ \pm}$and with $\widehat{U}^{\varepsilon}(\operatorname{cf}(7))$ in $\widehat{J}_{\alpha}$, it is easy to see that the difference $\widehat{u}^{\varepsilon}-\widehat{u}_{a p p}^{\varepsilon, k}$ satisfies an "appropriately scaled" Helmholtz equation in $\widehat{\Omega}$ with a source term (a right hand side) which is the sum of two terms. The first one has a support which coincides with the one of $\nabla \widehat{\Phi}_{\varepsilon}$, namely the overlaping zone and measures the defect of matching between $\widehat{U}^{\varepsilon, k}$ and $\widehat{u}_{ \pm}^{\varepsilon, k}$. It can be estimated as a function of $\varepsilon$ using the matching conditions (38), namely in

$$
O\left((\varepsilon|\log \varepsilon|)^{k-\frac{1}{2}}\right)
$$

The second term has a support which coincides with the one of $\widehat{\Phi}_{\varepsilon}$ and measures how much $\widehat{U}^{\varepsilon, k}$ fails to satisfy the Helmholtz equation exactly: it is shown (using the emdedded Laplace equations ) to be in $O\left(\varepsilon^{k}\right)$. Combining these "consistency" with a stability estimate (that is already used in the proof of theorem 1.2)) we get a global error estimate that can be optimized by choosing adequately $\varphi(\varepsilon)$, namely $\varphi(\varepsilon)=2 k \varepsilon|\log \varepsilon|$

$$
\sum_{ \pm}\left\|\widehat{u}_{ \pm}^{\varepsilon}-\widehat{u}_{a p p}^{\varepsilon, k}\right\|_{H^{1}\left(\widehat{\Omega}^{ \pm}\right)}+\left\|\widehat{U}^{\varepsilon}-\widehat{u}_{a p p}^{\varepsilon, k}\right\|_{H^{1}\left(\widehat{J}_{\alpha}\right)} \leqslant C_{k}(\varepsilon|\log \varepsilon|)^{k-1 / 2}
$$

To get the optimal "local" error estimate, we use the trick that:

$$
\widehat{u}_{ \pm}^{\varepsilon}-\widehat{u}_{ \pm}^{\varepsilon, k}=\left(\widehat{u}_{ \pm}^{\varepsilon}-\widehat{u}_{ \pm}^{\varepsilon, k+2}\right)+\left(\widehat{u}_{ \pm}^{\varepsilon, k+2}-\widehat{u}_{ \pm}^{\varepsilon, k}\right)
$$

Then, we remark that, when $\delta$ is fixed, for $\varepsilon$ small enough, $\widehat{u}_{ \pm}^{\varepsilon}-\widehat{u}_{a p p}^{\varepsilon, k}$ coincides with $\widehat{u}_{ \pm}^{\varepsilon}-\widehat{u}_{ \pm}^{\varepsilon, k}$ in $\widehat{\Omega}_{ \pm}^{\delta}$. Thus it can be estimated in

$$
O\left((\varepsilon|\log \varepsilon|)^{k+\frac{3}{2}}\right) \text {. }
$$

On the other hand the second term in (43) behaves for small $\varepsilon$ as $\varepsilon^{k+1} \widehat{u}_{ \pm}^{k}$. It is then easy to conclude. All the details are given in [2].

\section{The improved Kirchoff CONDitions}

\subsection{Construction and analysis of the improved Kirchhoff conditions}

The content of this section is based on the previous ones, but also on specific formulas that are established in the appendix B. To improve the transmission conditions satisfied by the limit problem, according to theorem 2.3 , it is natural to look at the transmission conditions satisfied by:

$$
u^{\varepsilon, 1}=u^{0}+\varepsilon \omega u^{1}
$$


For the Neumann jump condition, we first use (93) for $\mathrm{k}=1$, the fact that $U^{0}=u^{0}(0)$ in $\widehat{J}_{\alpha}$ and that the measure of $\widehat{J}_{\alpha}$ is $\tan \alpha$ to get

$$
\left[\frac{\partial u^{1}}{\partial s}\right]=-\omega \int_{\widehat{J}_{\alpha}} U^{0}=-\omega \tan \alpha u^{0}(0) \equiv-\omega \tan \alpha\left\langle u^{0}\right\rangle .
$$

Thus $u^{\varepsilon, 1}$ satisfies

$$
\left[\frac{\partial u^{\varepsilon, 1}}{\partial s}\right]=\left[\frac{\partial u^{0}}{\partial s}\right]+\varepsilon \omega\left[\frac{\partial u^{1}}{\partial s}\right]=-\varepsilon \omega^{2} \tan \alpha\left\langle u^{0}\right\rangle
$$

which can be rewritten, since $u^{0}=u^{\varepsilon, 1}-\varepsilon \omega u^{1}$

$$
\left[\frac{\partial u^{\varepsilon, 1}}{\partial s}\right]+\varepsilon \omega^{2} \tan \alpha\left\langle u^{\varepsilon, 1}\right\rangle=O\left(\varepsilon^{2}\right) .
$$

For the Dirichlet jump condition, we first use (95) for $\mathrm{k}=1$,

$$
\left[u^{1}\right]=\int_{\Sigma_{-}^{\alpha}} g_{-}^{0} \mathcal{W}_{\alpha}+\int_{\Sigma_{+}^{\alpha}} g_{+}^{0} \mathcal{W}_{\alpha} .
$$

with, using (92) for $k=1$,

Thus, by definition of $K(\alpha)$,

$$
g_{ \pm}^{0}:= \pm \frac{1}{\omega} \frac{\partial u_{ \pm}^{0}}{\partial s}(0) \equiv \pm \frac{1}{\omega}\left\langle\frac{\partial u^{0}}{\partial s}\right\rangle
$$

Therefore, $u^{\varepsilon, 1}$ satisfies

$$
\left[u^{1}\right]=\frac{1}{\omega} K(\alpha)\left\langle\frac{\partial u^{0}}{\partial s}\right\rangle
$$

which can be rewritten, using again $u^{0}=u^{\varepsilon, 1}-\varepsilon \omega u^{1}$

$$
\left[u^{\varepsilon, 1}\right]=\left[u^{0}\right]+\varepsilon \omega\left[u^{1}\right]=\varepsilon K(\alpha)\left\langle\frac{\partial u^{0}}{\partial s}\right\rangle
$$

$$
\left[u^{\varepsilon, 1}\right]-\varepsilon K(\alpha)\left\langle\frac{\partial u^{\varepsilon, 1}}{\partial s}\right\rangle=O\left(\varepsilon^{2}\right) .
$$

Finally, the transmission conditions for the 1D approximate solution $\tilde{u}^{\varepsilon}$, namely (17), are simply obtained by dropping the $O\left(\varepsilon^{2}\right)$ terms in (47) and (45) repectively.

Remark 3.1. The reader will immediately realize that the condition is only the first of a complete family of higher order conditions (this one being a priori the second order condition). These conditions are easily constructed by repeating the above construction for higher $k$ 's (see also point (iii) below). They have the same form than (17), except that the coefficients $\omega \tan \alpha$ and $K(\alpha) / \omega$ are replaced by "modified" coefficients. See [2] for more details.

We shall not give here a detailed proof of theorem 1.4 and error estimate (20) for the difference between $\tilde{u}^{\varepsilon}$ (or more precisely $\widehat{u}_{ \pm}^{\varepsilon, a p p}$ - $\left.\operatorname{cf}(18)\right)$ and $\widehat{u}_{ \pm}^{\varepsilon}$. Note however the result given by the error estimate (20) corresponds to a kind of "superconvergence result" with respect the expected $O\left(\varepsilon^{2}\right)$ accuracy. The main steps are the following:

(i) One identifies the terms of a 1D power series expansion of $\tilde{u}^{\varepsilon}$ :

$$
\tilde{u}^{\varepsilon}=\sum_{k=0}^{\infty}(\varepsilon \omega)^{k} \widetilde{u}^{k}(s)
$$


(ii) One proves rigourously (this is quite easy for a $1 \mathrm{D}$ problem) that

$$
\tilde{u}^{\varepsilon}-\left(\widetilde{u}^{0}+\varepsilon \omega \widetilde{u}^{1}+\cdots+(\varepsilon \omega)^{k} \widetilde{u}^{k}\right)=O\left(\varepsilon^{k+1}\right)
$$

(iii) One observes that $\widetilde{u}^{0}=u^{0}, \quad \widetilde{u}^{1}=u^{1}$ and $\widetilde{u}^{2}=u^{2}$, the last equality being an unexpected bonus linked to the fact that $\widehat{J}_{\alpha}$ has an symmetry axis. Indeed, (87) for $k=1$ gives

$$
\frac{\partial U^{1}}{\partial n}+T_{ \pm} U^{1}= \pm \frac{1}{\omega} \frac{\partial u_{ \pm}^{0}}{\partial s}(0) \equiv \pm \frac{1}{\omega}\left\langle\frac{\partial u^{0}}{\partial s}\right\rangle
$$

So, using the definition of $\mathcal{W}_{\alpha}$ (cf. $(11,(10))$ and (96) for $k=1$, we conclude via lemma B.2 that

$$
U^{1}=\left\langle u^{1}\right\rangle+\frac{1}{\omega}\left\langle\frac{\partial u^{0}}{\partial s}\right\rangle(0) \mathcal{W}_{\alpha}
$$

Thus, writing (93) for $\mathrm{k}=2$ we get

$$
\left[\frac{\partial u^{2}}{\partial s}\right]=-\omega \int_{\widehat{J}_{\alpha}} U^{1}=-\omega \tan \alpha\left\langle u^{1}\right\rangle
$$

which is identical to (44) after index shifting. On the other hand, writing (95) for $\mathrm{k}=2$ gives

$$
\left[u^{2}\right]=\int_{\Sigma_{-}^{\alpha}} g_{-}^{1} \mathcal{W}_{\alpha}+\int_{\Sigma_{+}^{\alpha}} g_{+}^{1} \mathcal{W}_{\alpha}
$$

where, using $(92)$ for $k=1$ and $\delta_{p}^{1}=0, g_{ \pm}^{1}:= \pm \frac{1}{\omega} \frac{\partial u_{ \pm}^{1}}{\partial s}(0)$. Thus

$$
\left[u^{2}\right]=\frac{1}{\omega}\left(\frac{\partial u_{+}^{1}}{\partial s}(0) \int_{\Sigma_{+}^{\alpha}} \mathcal{W}_{\alpha}-\frac{\partial u_{-}^{1}}{\partial s}(0) \int_{\Sigma_{-}^{\alpha}} \mathcal{W}_{\alpha}\right)
$$

Since $\widehat{J}_{\alpha}$ has an axis of symmetry, $\mathcal{W}_{\alpha}$ is anti-symmetric with respect to this line and consequently

$$
\left.\int_{\Sigma_{+}^{\alpha}} \mathcal{W}_{\alpha}=-\int_{\Sigma_{-}^{\alpha}} \mathcal{W}_{\alpha}=\frac{K(\alpha)}{2} . \quad \text { (using the definition }(14) \text { of } K(\alpha)\right)
$$

Finally, we have

$$
\left[u^{2}\right]=\frac{1}{\omega} K(\alpha)\left\langle\frac{\partial u^{1}}{\partial s}\right\rangle .
$$

which is nothing but (46) after index shifting.

(iv) This allows us to write in $\widehat{\Omega}_{ \pm}^{\delta}$ (modulo some abuse of notation)

$$
\widehat{u}_{ \pm}^{\varepsilon}-\tilde{u}^{\varepsilon}=\left(\widehat{u}_{ \pm}^{\varepsilon}-\widehat{u}_{ \pm}^{\varepsilon, 2}\right)+\left(\widetilde{u}^{0}+\varepsilon \omega \widetilde{u}^{1}+(\varepsilon \omega)^{2} \widetilde{u}^{2}-\tilde{u}^{\varepsilon}\right)
$$

and one concludes using (48) for $k=2$, (41) and the triangular inequality. 


\subsection{Reflection and transmission of waves with the improved conditions}

We consider here the case of the junction of two semi-infinite strips, i.e. $L_{ \pm}=+\infty$ and look at the approximate model, i.e. the $1 \mathrm{D}$ Hemlholtz equation for $\pm s>0$ with the transmission conditions (17) at $s=0$. We are more precisely interested in the reflection-transmission of an incident wave in the domain $s<0$, i. e. look for a solution of the form

$$
\tilde{u}^{\varepsilon}(s)=\exp (\imath \omega s)+R_{\varepsilon}(\alpha, \omega) \exp (-\imath \omega s) \quad \text { for } s<0, \quad \tilde{u}^{\varepsilon}(s)=T_{\varepsilon}(\alpha, \omega) \exp (\imath \omega s) \quad \text { for } s>0
$$

where the complex numbers $R_{\varepsilon}(\alpha, \omega)$ and $T_{\varepsilon}(\alpha, \omega)$ are respectively the reflection coefficients. Substituting (51) into (17) leads to a $2 \times 2$ linear system for $\left(R_{\varepsilon}(\alpha, \omega), T_{\varepsilon}(\alpha, \omega)\right)$ whose explicit solution is given by

$$
\left\{\begin{array}{l}
R_{\varepsilon}(\alpha, \omega)=\frac{1}{2}\left(\frac{1+\imath \varepsilon \omega \tan \alpha / 2}{1-\imath \varepsilon \omega \tan \alpha / 2}-\frac{1+\imath \varepsilon \omega K(\alpha) / 2}{1-\imath \varepsilon \omega K(\alpha) / 2}\right) \\
T_{\varepsilon}(\alpha, \omega)=\frac{1}{2}\left(\frac{1+\imath \varepsilon \omega \tan \alpha / 2}{1-\imath \varepsilon \omega \tan \alpha / 2}+\frac{1+\imath \varepsilon \omega K(\alpha) / 2}{1-\imath \varepsilon \omega K(\alpha) / 2}\right)
\end{array}\right.
$$

In particular, for small values of $\varepsilon \omega$, we have

$$
\left\{\begin{array}{rlrl}
R_{\varepsilon}(\alpha, \omega) & =\imath \varepsilon \omega r_{1}(\alpha)+O\left((\varepsilon \omega)^{2}\right), & & r_{1}(\alpha)=\frac{1}{2}(\tan \alpha-K(\alpha)), \\
T_{\varepsilon}(\alpha, \omega)=1+\imath \varepsilon \omega t_{1}(\alpha)+O\left((\varepsilon \omega)^{2}\right), & t_{1}(\alpha)=\frac{1}{2}(\tan \alpha+K(\alpha)) .
\end{array}\right.
$$

We comment below the formulas (52) and (53) using in particular lemma 1.3:

- Formulas (52) show that the coefficients $R_{\varepsilon}(\alpha, \omega)$ and $T_{\varepsilon}(\alpha, \omega)$ tend respectively to 0 and 1 when $\varepsilon$ tends to 0 , which was expected from the limit problem (4).

- The property (15) implies that in particular $K(\alpha)$ tends to 0 when $\alpha$ tends to 0 and formulas (52) allow us to check that, as the intuition suggests, $R_{\varepsilon}(\alpha, \omega)$ and $T_{\varepsilon}(\alpha, \omega)$ to 0 and 1 when $\alpha$ tends to 0 . One even has

$$
R_{\varepsilon}(\alpha, \omega)=O\left(\alpha^{2}\right), \quad T_{\varepsilon}(\alpha, \omega)=1+O\left(\alpha^{2}\right) .
$$

- A consequence of (53) is that

$$
\left|R_{\varepsilon}(\alpha, \omega)\right|=O(\varepsilon), \quad\left|T_{\varepsilon}(\alpha, \omega)\right|=1+O\left(\varepsilon^{2}\right)
$$

which means that in practice, the reflection phenomenon is in amplitude more directly visible than the transmission phenomenon.

- For small values of $\varepsilon$, the formulas (53) show that in first approximation, $R_{\varepsilon}(\alpha, \omega)$ and $1-T_{\varepsilon}(\alpha, \omega)$ are proportional to $r_{1}(\alpha)$ and $t_{1}(\alpha)$ respectively. The monotonicity result of lemma 1.3 implies in particular that $r_{1}(\alpha)$ is positive and increasing with $\alpha$. This is coherent with the intuition which suggests that the amplitude of the reflected wave should increase with $\alpha$. This is illustrated numerically by figures 6 and 7 .

- The fact that, for small values of $\varepsilon, R_{\varepsilon}(\alpha, \omega)$ is ( as $1-T_{\varepsilon}(\alpha, \omega)$ ) also proportional to $\imath \omega$ means that, going back to the time domain and the wave equation, the reflection phenomenon corresponds in first approximation to a derivation with respect to time of the incident signal. This will be illustrated in the next section with numerical simulations. 
Figures 6 and $7:\left|T_{\varepsilon}(\alpha, \omega)\right|$ and $\left|R_{\varepsilon}(\alpha, \omega)\right|$ as functions of $\alpha$

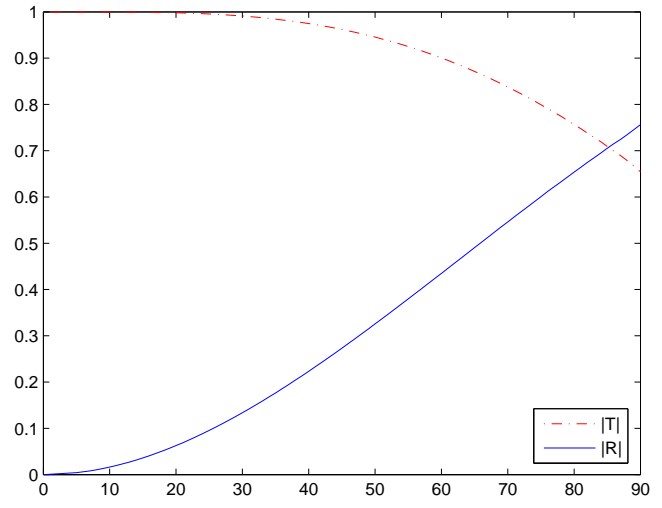

Figure 6. $\varepsilon \omega=1$

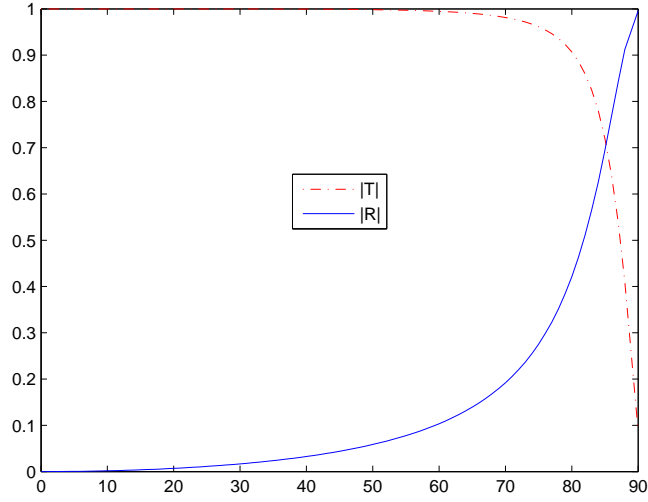

Figure 7. $\varepsilon \omega=0.1$

\subsection{Numerical validation of the improved conditions}

In this section, we go back to the time domain ( $t$ will denote the time) and the wave equation on which it is easier to illustrate out results. The approximate 1D problem we consider is

$$
\begin{cases}\frac{\partial^{2} \tilde{u}^{\varepsilon}}{\partial t^{2}}-\frac{\partial^{2} \tilde{u}^{\varepsilon}}{\partial s^{2}}=0, & \text { in } \mathbb{R}_{+} \times(]-L^{-}, 0[\cup] 0, L^{+}[) \\ {\left[\tilde{u}^{\varepsilon}\right]-\varepsilon K(\alpha)\left\langle\frac{\partial \tilde{u}^{\varepsilon}}{\partial s}\right\rangle=0, \quad\left[\frac{\partial \tilde{u}^{\varepsilon}}{\partial s}\right]-\varepsilon \tan \alpha\left\langle\frac{\partial^{2} \tilde{u}^{\varepsilon}}{\partial t^{2}}\right\rangle=0,} & \text { at } s=0 .\end{cases}
$$

For the source term, we consider initial conditions chosen in such a way that for initial values of $t$ the solution corresponds to an incident wave in $s<0$

$$
\tilde{u}^{\varepsilon}(s, t)=u_{0}(s-t) \quad\left(\Longleftrightarrow \tilde{u}^{\varepsilon}(s, 0)=u_{0}(s), \quad \frac{\partial \tilde{u}^{\varepsilon}}{\partial t}(s, 0)=-u_{0}^{\prime}(s)\right)
$$

where $u_{0}$ is a smooth "Gaussian like" function compactly supported in $s<0$. For the exact or reference solution, we consider the wave equation in the domain $\Omega^{\varepsilon}$ with $L_{ \pm}=+\infty$ and Neumann boundary conditions and with the "same initial conditions" (we mean here that the initial data are independent of the transverse coordinate in the slot).

\subsubsection{Numerical results on the $2 D$ exact solution}

We used the code MONTJOIE ${ }^{2}$ to compute the $2 \mathrm{D}$ exact solution. We plot on figures 8 to 10 the solution at a time $t$ large enough to see the effects of the junction. We take $\varepsilon=\lambda / 10$ (where $\lambda$ is the wave length of the Cauchy data).

On the figures 8 to 10 , we plot the solution on the scaled set $\widehat{\Omega}_{-} \cup \widehat{J}_{\alpha} \cup \widehat{\Omega}_{-}$, for differents values of $\alpha$, and we use the same range to see the effect of the junction on the reflection (the green color corresponds to the value $0)$. We can see about the reflection wave that :

- The form is the derivate of the "Gaussian like" function

- Its amplitude increases with the value of $\alpha$

\footnotetext{
2 url: http://www-rocq.inria.fr/poems/montjoie/
} 
Figures 8 to $10: 2 \mathrm{D}$ Computations of the exact solution $(\varepsilon=0.1 \lambda)$.

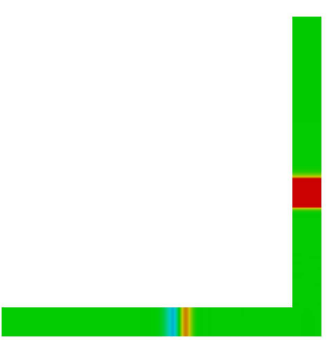

FiguRE 8. $\alpha=\frac{\pi}{4}$

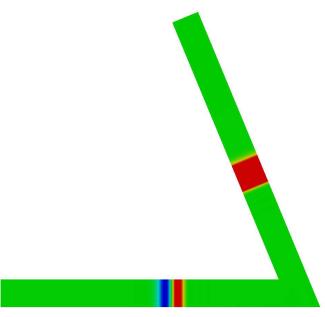

Figure $9 . \quad \alpha=\frac{5 \pi}{16}$

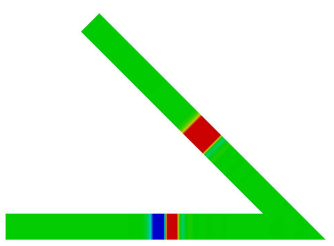

FiguRE 10. $\alpha=\frac{3 \pi}{8}$

\subsubsection{Numerical results on the improved $1 D$ model}

For the 1D model (56), we saw in the section 3.2 that, for small values of $\alpha \varepsilon / \lambda$, the reflection phenomen corresponds in first approximation to a derivation with respect to time of the incident signal. To see this phenomena, we compute the function $\tilde{u}^{\varepsilon}$ solution of (56) with the initial data $u_{0}(s)=\exp \left(-5(s+L / 2)^{2}\right)$, and we plot $\varepsilon^{-1} \tilde{u}^{\varepsilon}(t, s)$ as function of $t$, with $s=-3 L / 4$, for differents values of $\varepsilon$, and we compared with the translation of $-(\tan (\alpha)-K(\alpha)) u^{\prime}(s)$

Figures 11 and $12:$ plot of $\varepsilon^{-1} \tilde{u}^{\varepsilon}(t, s)$ as function of $t$, with $s=-\frac{3 L}{4}$

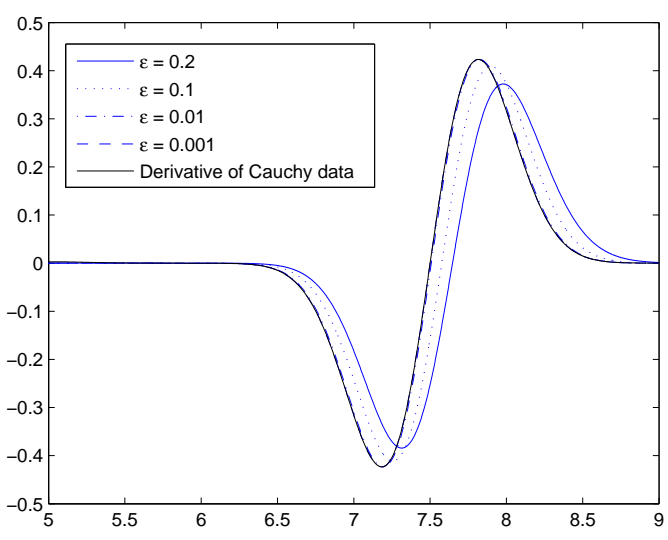

FiguRE 11. $\alpha=\frac{\pi}{4}$

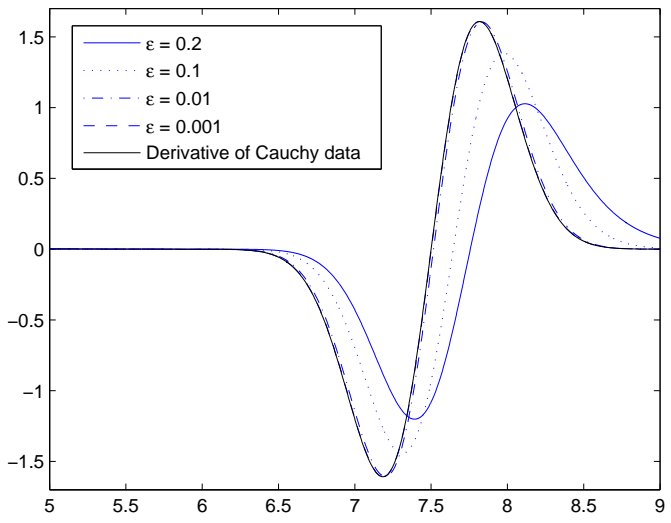

FIGURE 12. $\alpha=\frac{3 \pi}{8}$

We can see in figures 11 and 12 that our comments about the reflection phenomena are numerically discernible.

\subsubsection{Convergence between the approximated model and the exact model}

We wish to check numerically the error (20) (theorem 1.4). We take $\delta=0.2, \alpha=\frac{\pi}{4}$, and proceed as follow :

(i) We compute $K(\alpha)$ once for all with a FreeFem $++^{3}$ script.

(ii) For differents values of $\varepsilon \in] 10^{-3}, 10^{-1}$ [, we compute $u^{\varepsilon}$ solution of $(3)$ and $\tilde{u}^{\varepsilon}$ solution of $(16,17)$, and we build $\widehat{u}_{ \pm}^{\varepsilon, a p p}$ by (18)

(iii) We compute $\sum_{ \pm}\left\|\widehat{u}_{ \pm}^{\varepsilon, a p p}-\widehat{u}_{ \pm}^{\varepsilon}\right\|_{\mathrm{H}^{1}\left(\widehat{\Omega}_{ \pm}^{\delta}\right)}$

\footnotetext{
${ }^{3} \mathrm{url}:$ http://www.freefem.org/ff $++/$
} 


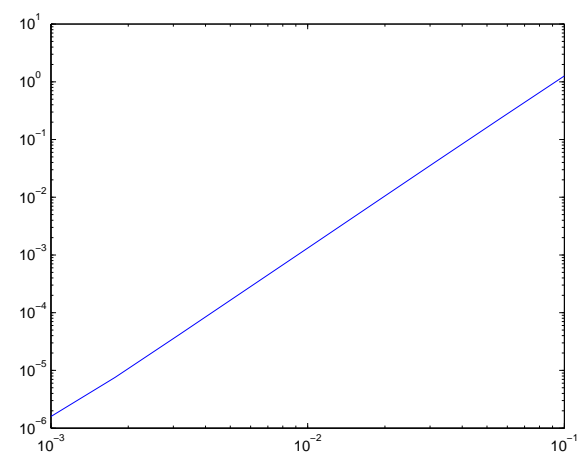

FiguRE 13. $\sum_{ \pm}\left\|\widehat{u}_{ \pm}^{\varepsilon, a p p}-\widehat{u}_{ \pm}^{\varepsilon}\right\|_{\mathrm{H}^{1}\left(\widehat{\Omega}_{ \pm}^{\delta}\right)}$ as function of $\varepsilon$ (logscale) - the slope is equal to 2.9497 We can see on figure 13 that the error $\sum_{ \pm}\left\|\widehat{u}_{ \pm}^{\varepsilon, a p p}-\widehat{u}_{ \pm}^{\varepsilon}\right\|_{\mathrm{H}^{1}\left(\widehat{\Omega}_{ \pm}^{\delta}\right)}$ behaves as $C \varepsilon^{\beta}$, with $\beta \simeq 3$, as expected.

\section{A. Derivation of the matching Conditions}

It is first useful to describe the form of the functions $U^{k}$ 's in the two semi-strips $\widehat{B}_{ \pm}$(see figure 5 ): this is the object of the next section.

\section{A.1. Modal expansion of solutions of embedded Laplace equations}

Let consider $U_{ \pm}^{k}: \widehat{B}_{ \pm} \mapsto \mathbb{C}, \quad k \geqslant 0$, in $\mathrm{H}_{l o c}^{1}\left(\widehat{B}_{ \pm}\right)$satisfying

$$
\Delta U_{ \pm}^{0}=0, \quad \Delta U_{ \pm}^{1}=0, \quad \Delta U_{ \pm}^{k}+U_{ \pm}^{k-2}=0, \quad \text { in } \widehat{B}_{ \pm}, \quad \frac{\partial U_{ \pm}^{k}}{\partial \widehat{\nu}}=0 \quad \text { for } \widehat{\nu}=-1,0, \quad k \geqslant 2
$$

Later, in section A.2, the results of the present section will be applied to $U_{ \pm}^{k}$ the respective restrictions of $U^{k}$, the coefficients in the expansion (29). We shall use the local coordinates $(\widehat{s}, \widehat{\nu})=\left(\widehat{\mathbf{x}} \cdot \mathbf{t}^{ \pm}, \widehat{\mathbf{x}} \cdot \mathbf{n}^{ \pm}\right)$such that:

$$
\left.\widehat{\mathbf{x}} \in \widehat{B}_{ \pm} \quad \Longleftrightarrow \quad( \pm \widehat{s}, \widehat{\nu}) \in\right] 0, \infty[\times]-1,0[
$$

The behaviour of the fields $U^{k}$ in the two semi-strips $\widehat{B}_{ \pm}$is easily described using separation of variables in $(\widehat{s}, \widehat{\nu})$ coordinates, which naturally introduces the basis $w_{p}(\widehat{\nu})$ (cf. (13)), which are adapted to the Neumann conditions at $\widehat{\nu}=-1$ or 0 : there exists $1 \mathrm{D}$ functions $U_{ \pm, p}^{k}(\widehat{s})$ such that

$$
U_{ \pm}^{k}(\widehat{s}, \widehat{\nu})=\sum U_{ \pm, p}^{k}(\widehat{s}) w_{p}(\widehat{\nu})
$$

If we substitute formally (59) into the equations (57), we obtain, according to remark 2.1 :

$$
\forall k \in \mathbb{N}, \forall p \in \mathbb{N}, \quad \frac{\partial^{2} U_{ \pm, p}^{k}}{\partial \widehat{s}^{2}}-p^{2} \pi^{2} U_{ \pm, p}^{k}+U_{ \pm, p}^{k-2}=0
$$

The resolution of (60) is a tedious but simple exercise on ordinary differential equations. In what follows, we reproduce some results of [3], that we present in a slightly different form, more adapted to the purpose of this paper. After having remarked that the change of unknown

$$
U_{ \pm, p}^{k}=\exp ( \pm \pi \widehat{s}) V_{ \pm, p}^{k}
$$


leads to the equations (with the convention $V_{ \pm, p}^{k} \equiv 0$ for $k<0$ )

$$
\frac{\partial^{2} V_{ \pm, p}^{k}}{\partial \widehat{s}^{2}} \pm 2 p \pi \frac{\partial V_{ \pm, p}^{k}}{\partial \widehat{s}}=-V_{ \pm, p}^{k-2}
$$

we introduce, for each $p \in \mathbb{N}$, two sequences of polynomials solutions of (61)

$$
\left(c_{ \pm, p}^{k}(\widehat{s}), d_{ \pm, p}^{k}(\widehat{s})\right), \quad k \in \mathbb{N},
$$

which are defined inductively on $k$, for each $p \in \mathbb{N}$ and are identically 0 for odd values of $k$.

- The value $p=0$ plays a particular role, since equation (60) degenerates. For $k=0,1$ one has

$$
c_{ \pm, 0}^{0}(\widehat{s})=\imath \widehat{s}, \quad c_{ \pm, 0}^{1}(\widehat{s})=0, \quad d_{ \pm, 0}^{0}(\widehat{s})=1, \quad d_{ \pm, 0}^{1}(\widehat{s})=0,
$$

continuing for $k \geqslant 2$ with:

$$
\mid \begin{array}{ll}
\frac{\partial^{2} c_{ \pm, 0}^{k}}{\partial \widehat{s}^{2}}=-c_{ \pm, 0}^{k-2}, & c_{ \pm, 0}^{k}(0)=\frac{\partial c_{ \pm, 0}^{k}}{\partial \widehat{s}}(0)=0 \\
\frac{\partial^{2} d_{ \pm, 0}^{k}}{\partial \widehat{s}^{2}}=-d_{ \pm, 0}^{k-2}, & d_{ \pm, 0}^{k}(0)=\frac{\partial d_{ \pm, 0}^{k}}{\partial \widehat{s}}(0)=0
\end{array}
$$

It is easy to see that, for even $k$ 's, one recovers the monomials of the series expansion of $\exp (\imath \widehat{s})$ :

$$
\widehat{c}_{ \pm, 0}^{2 m}(\widehat{s})=\frac{(\imath \widehat{s})^{2 m+1}}{(2 m+1) !}, \quad \widehat{d}_{ \pm, 0}^{2 m}(\widehat{s})=\frac{(\imath \widehat{s})^{2 m}}{(2 m) !}
$$

- For $p \geqslant 1$, one starts from

$$
c_{ \pm, p}^{0}(\widehat{s})=1, \quad c_{ \pm, p}^{1}(\widehat{s})=0 \text { for } p \geqslant 0 ; \quad d_{ \pm, p}^{0}(\widehat{s})=1, \quad d_{ \pm, p}^{1}(\widehat{s})=0 . \text { for } p \geqslant 0,
$$

Then, $\left(c_{ \pm, p}^{k}(\widehat{s}), d_{ \pm, p}^{k}(\widehat{s})\right)$ are defined as the unique polynomial solutions of

$$
\mid \begin{array}{ll}
\frac{\partial^{2} c_{ \pm, p}^{k}}{\partial \widehat{s}^{2}} \pm 2 p \pi \frac{\partial c_{ \pm, p}^{k}}{\partial \widehat{s}}=-c_{ \pm, p}^{k-2}, & c_{ \pm, p}^{k}(0)=0 \\
\frac{\partial^{2} d_{ \pm, p}^{k}}{\partial \widehat{s}^{2}} \mp 2 p \pi \frac{\partial d_{ \pm, p}^{k}}{\partial \widehat{s}}=-d_{ \pm, p}^{k-2}, & d_{ \pm, p}^{k}(0)=0
\end{array}
$$

By induction on $k$, it is easy to see that,

$$
c_{ \pm, p}^{2 k} \text { has degree } k, \quad d_{ \pm, p}^{2 k} \text { has degree } k, \quad c_{ \pm, p}^{2 k+1}=d_{ \pm, p}^{2 k+1} \equiv 0 .
$$

Next, we construct two families of functions $\mathbf{c}_{ \pm, p}^{k}$ and $\mathbf{d}_{ \pm, p}^{k}$ from $\widehat{\Omega}_{ \pm}$into $\mathbb{C}$, for $p \in \mathbb{N}$ and $k \in \mathbb{N}^{*}$, by:

$$
\mid \begin{aligned}
& \mathbf{c}_{ \pm, p}^{k}(\widehat{\mathbf{x}})=\exp ( \pm p \pi \widehat{s}) c_{ \pm, p}^{k}(\widehat{s}) w_{p}(\widehat{\nu}) \\
& \mathbf{d}_{ \pm, p}^{k}(\widehat{\mathbf{x}})=\exp (\mp p \pi \widehat{s}) d_{ \pm, p}^{k}(\widehat{s}) w_{p}(\widehat{\nu})
\end{aligned}
$$


that constitute particular families of embedded Laplace's equations:

$$
\forall p \in \mathbb{N}, \quad \forall k \in \mathbb{N}, \quad \Delta \mathbf{c}_{ \pm, p}^{k}=-\mathbf{c}_{ \pm, p}^{k-2}, \quad \Delta \mathbf{d}_{ \pm, p}^{k}=-\mathbf{d}_{ \pm, p}^{k-2}, \quad \text { in } \widehat{\Omega}_{ \pm}
$$

with homogeneous Neumann boundary conditions at $\widehat{\nu}=-1,0$. The interest of the introduction of the functions $\mathbf{c}_{ \pm, p}^{k}, \mathbf{d}_{ \pm, p}^{k}$ clearly appers in the following result :

Lemma A.1. Let $\left\{U_{ \pm}^{k} \in H_{l o c}^{1}\left(\widehat{B}_{ \pm}\right), k \geqslant 0\right\}$ satisfying (57), there exists two sequences $\left(\gamma_{p, k}^{ \pm}\right)_{(p, k) \in \mathbb{N}^{2}}$ and $\left(\delta_{p, k}^{ \pm}\right)_{(p, k) \in \mathbb{N}^{2}}$ of complex numbers such as

$$
U_{ \pm}^{k}=\sum_{m=0}^{k} \sum_{p=0}^{\infty}\left(\gamma_{p, k-m}^{ \pm} \mathbf{c}_{ \pm, p}^{m}+\delta_{p, k-m}^{ \pm} \mathbf{d}_{ \pm, p}^{m}\right), \quad\left(\text { in } \mathrm{H}_{l o c}^{1}\left(\widehat{B}_{ \pm}\right)\right)
$$

Proof. It is indentical to the proof of lemma 3.1 of [3].

We can formalize this result by introducing the vector spaces

$$
\mathcal{V}_{k}\left(\widehat{B}_{ \pm}\right)=\operatorname{span}\left\{\left(\mathbf{c}_{ \pm, p}^{m}, \mathbf{d}_{ \pm, p}^{m}\right), \quad p \in \mathbb{N}, \quad m \leqslant k\right\}, \quad \mathcal{V}\left(\widehat{B}_{ \pm}\right)=\bigcup_{k=0}^{\infty} \mathcal{V}_{k}\left(\widehat{B}_{ \pm}\right)
$$

and the linear forms $\mathcal{U}_{ \pm} \in \mathcal{V}\left(\widehat{B}_{ \pm}\right) \rightarrow \mathcal{N}_{p, m}^{ \pm}\left(\mathcal{U}_{ \pm}\right) \in \mathbb{C}$ and $\mathcal{U}_{ \pm} \in \mathcal{V}\left(\widehat{B}_{ \pm}\right) \rightarrow \mathcal{D}_{p, m}^{ \pm}\left(\mathcal{U}_{ \pm}\right) \in \mathbb{C}$ such that

$$
\forall \mathcal{U}_{ \pm} \in \mathcal{V}_{k}\left(\widehat{B}_{ \pm}\right), \quad \mathcal{U}_{ \pm}=\sum_{m=0}^{k} \sum_{p=0}^{\infty}\left(\mathcal{N}_{p, m}^{ \pm}\left(\mathcal{U}_{ \pm}\right) \mathbf{c}_{ \pm, p}^{m}+\mathcal{D}_{p, m}^{ \pm}\left(\mathcal{U}_{ \pm}\right) \mathbf{d}_{ \pm, p}^{m}\right)
$$

In the following, the value $m=0$ will play a particular role and that is why we shall denote

$$
\forall \mathcal{U}_{ \pm} \in \mathcal{V}\left(\widehat{B}_{ \pm}\right), \quad \mathcal{N}_{p}^{ \pm}\left(\mathcal{U}_{ \pm}\right):=\mathcal{N}_{p, 0}^{ \pm}\left(\mathcal{U}_{ \pm}\right), \quad \mathcal{D}_{p}^{ \pm}\left(\mathcal{U}_{ \pm}\right):=\mathcal{D}_{p, 0}^{ \pm}\left(\mathcal{U}_{ \pm}\right)
$$

With this notation, lemma A.1 can be reinterpreted as

Corollary A.2. Let $\left\{U_{ \pm}^{k} \in H_{l o c}^{1}\left(\widehat{B}_{ \pm}\right), k \geqslant 0\right\}$ satisfying (57), then

$$
U_{ \pm}^{k} \in \mathcal{V}_{k}\left(\widehat{B}_{ \pm}\right) \quad \text { and } \quad \mathcal{N}_{p, m}^{ \pm}\left(U_{ \pm}^{k}\right)=\mathcal{N}_{p}^{ \pm}\left(U_{ \pm}^{k-m}\right), \quad \mathcal{D}_{p, m}^{ \pm}\left(U_{ \pm}^{k}\right)=\mathcal{D}_{p}^{ \pm}\left(U_{ \pm}^{k-m}\right)
$$

and consequently,

$$
U_{ \pm}^{k}=\sum_{m=0}^{k} \sum_{p=0}^{\infty}\left(\mathcal{N}_{p}^{ \pm}\left(U_{ \pm}^{k-m}\right) \mathbf{c}_{ \pm, p}^{m}+\mathcal{D}_{p}^{ \pm}\left(U_{ \pm}^{k-m}\right) \mathbf{d}_{ \pm, p}^{m}\right), \quad\left(\text { in } \mathrm{H}_{l o c}^{1}\left(\widehat{B}_{ \pm}\right)\right)
$$

We can give a more tractable definition of $\mathcal{N}_{0}^{ \pm}$and $\mathcal{D}_{0}^{ \pm}$that can be interpreted as "mean" Dirichlet or Neumann trace operators at $\widehat{s}=0$ (i.e. on $\Sigma_{ \pm}^{\alpha}$ ):

\section{Lemma A.3.}

$$
\forall \mathcal{U}_{ \pm} \in \mathcal{V}\left(\widehat{B}_{ \pm}\right), \quad \mathcal{D}_{0}^{ \pm}\left(\mathcal{U}_{ \pm}\right)=\int_{0}^{1} \mathcal{U}_{ \pm}(0, \widehat{\nu}) d \widehat{\nu}, \quad \mathcal{N}_{0}^{ \pm}\left(\mathcal{U}_{ \pm}\right)=\frac{1}{\imath} \int_{0}^{1} \frac{\partial \mathcal{U}_{ \pm}}{\partial \widehat{s}}(0, \widehat{\nu}) d \widehat{\nu}
$$


Proof. Integrating (72) with respect to $\widehat{\nu}$ gives:

$$
\forall \mathcal{U}_{ \pm} \in \mathcal{V}_{k}\left(\widehat{B}_{ \pm}\right), \quad \int_{0}^{1} \mathcal{U}_{ \pm}(\widehat{s}, \widehat{\nu}) d \widehat{\nu}=\sum_{m=0}^{k}\left(\mathcal{N}_{0, m}^{ \pm}\left(\mathcal{U}_{ \pm}\right) c_{ \pm, 0}^{m}(\widehat{s})+\mathcal{D}_{0, m}^{ \pm}\left(\mathcal{U}^{ \pm}\right) d_{ \pm, 0}^{m}(\widehat{s})\right)
$$

For $\widehat{s}=0$, this equality leads to the first equality of (76), thanks to (62), (63) and (73). For the second equality, we first differentiate (77) with respect to $\widehat{s}$ and take $\widehat{s}=0$ (using again (62), (63) and (73)) .

Moreover, for functions which belong to the kernel of the linear forms $\mathcal{N}_{p}^{ \pm}$for $p>1$, we have

Lemma A.4. Let $\mathcal{V}_{0}\left(\widehat{B}_{ \pm}\right):=\left\{\mathcal{U}_{ \pm} \in \mathcal{V}\left(\widehat{B}_{ \pm}\right) / \forall p>1, \quad \mathcal{N}_{p}^{ \pm}\left(\mathcal{U}_{ \pm}\right)=0\right\}$. Then

$$
\forall \mathcal{U} \in \mathcal{V}_{0}\left(\widehat{B}_{ \pm}\right), \quad \mathcal{D}_{p}^{ \pm}\left(\mathcal{U}_{ \pm}\right)=\int_{0}^{1} \mathcal{U}_{ \pm}(0, \widehat{\nu}) w_{p}(\widehat{\nu}) d \widehat{\nu}
$$

Proof. It is similar to the proof of lemma A.3, except that we first multiply (72) by $w_{p}$ before integrating with respect to $\widehat{\nu}$ and then use (65) and (66). The details are left to the reader.

\section{A.2. Derivation of the matching conditions}

We denote respectively $\mathcal{L}$ and $\mathcal{R}$ the left and right hand side of (36). To get another expression for $\mathcal{R}$, we use the Taylor series expansion (in $s$ ) of each $u_{ \pm}^{k}$

$$
\mathcal{R}=\sum_{k=0}^{\infty} \sum_{m=0}^{\infty}(\varepsilon \omega)^{k} s^{m} \frac{1}{m !} \frac{\partial^{m} u_{ \pm}^{k}}{\partial s^{m}}(0)+o(\varepsilon \omega)^{\infty}
$$

For $\mathcal{L}$, we use the expansion (75) for $U_{ \pm}^{k}$ and obtain (using $\sum_{k=0}^{\infty} \sum_{m=0}^{k} \equiv \sum_{m=0}^{\infty} \sum_{k=m}^{\infty}$ )

$$
\mathcal{L}=\sum_{m=0}^{\infty} \sum_{p=0}^{\infty} \sum_{k=m}^{\infty}(\varepsilon \omega)^{k}\left(\mathcal{N}_{p}^{ \pm}\left(U_{ \pm}^{k-m}\right) \mathbf{c}_{ \pm, p}^{m}(s / \varepsilon, \widehat{\nu} / \varepsilon)+\mathcal{D}_{p}^{ \pm}\left(U_{ \pm}^{k-m}\right) \mathbf{d}_{ \pm, p}^{m}(s / \varepsilon, \widehat{\nu} / \varepsilon)\right)+o(\varepsilon \omega)^{\infty}
$$

Since for $p \geqslant 1$, the functions $\mathbf{d}_{ \pm, p}^{m}$ are exponentially decreasing at infinity and since the functions $\varphi$ belong to the class $\mathcal{C}$ (see (21)), the corresponding terms in the previous sum can be "put into" the $o(\varepsilon \omega)^{\infty}$ part. For the rest of the sum, we distinguish the terms for $p=0$, for which we use the formulas (64) from the terms corresponding to $p \geqslant 1$ (which are exponentially increasing at infinity):

$$
\begin{aligned}
\mathcal{L} & =\sum_{m^{\prime}=0}^{\infty} \sum_{k=2 m^{\prime}}^{\infty}(\varepsilon \omega)^{k} \mathcal{N}_{0}^{ \pm}\left(U_{ \pm}^{k-2 m^{\prime}}\right) \frac{(\imath s / \varepsilon)^{2 m^{\prime}+1}}{\left(2 m^{\prime}+1\right) !}+\sum_{m^{\prime}=0}^{\infty} \sum_{k=2 m^{\prime}}^{\infty}(\varepsilon \omega)^{k} \mathcal{D}_{0}^{ \pm}\left(U_{ \pm}^{k-2 m^{\prime}}\right) \frac{(\imath s / \varepsilon)^{2 m^{\prime}}}{\left(2 m^{\prime}\right) !} \\
& +\sum_{m=0}^{\infty} \sum_{p=1}^{\infty} \sum_{k=m}^{\infty}(\varepsilon \omega)^{k} \mathcal{N}_{p}^{ \pm}\left(U_{ \pm}^{k-m}\right) \mathbf{c}_{ \pm, p}^{m}(s / \varepsilon, \widehat{\nu} / \varepsilon)+o(\varepsilon \omega)^{\infty} .
\end{aligned}
$$

Using the change of index $k \rightarrow k+2 m^{\prime}+1$ in the first sum (resp. $k \rightarrow k+2 m^{\prime}$ in the second one), we get

$$
\begin{aligned}
\mathcal{L} & =\sum_{m^{\prime}=0}^{\infty} \sum_{k=-1}^{\infty}(\varepsilon \omega)^{k} \mathcal{N}_{0}^{ \pm}\left(U_{ \pm}^{k+1}\right) \frac{(\imath \omega s)^{2 m^{\prime}+1}}{\left(2 m^{\prime}+1\right) !}+\sum_{m^{\prime}=0}^{\infty} \sum_{k=0}^{\infty}(\varepsilon \omega)^{k} \mathcal{D}_{0}^{ \pm}\left(U_{k}\right) \frac{(\imath s \omega)^{2 m^{\prime}}}{\left(2 m^{\prime}\right) !} \\
& +\sum_{m=0}^{\infty} \sum_{p=1}^{\infty} \sum_{k=m}^{\infty}(\varepsilon \omega)^{k} \mathcal{N}_{p}^{ \pm}\left(U_{ \pm}^{k-m}\right) \mathbf{c}_{ \pm, p}^{m}(s / \varepsilon, \widehat{\nu} / \varepsilon)+o(\varepsilon \omega)^{\infty}
\end{aligned}
$$


Finally, the formal identification of the expressions (80) and (79) in the overlaping zone $\mathcal{O}_{ \pm}(\varepsilon)$, as functions of $s$ and $\varepsilon$, will lead us to our matching conditions.

First, for $p \geqslant 1$, after multiplication of (80) and (79) by $w_{p}(\widehat{\nu})$ and integration over $\widehat{\nu}$, we get

$$
\sum_{m=0}^{\infty} c_{ \pm, p}^{m}(s / \varepsilon)\left(\sum_{k=m}^{\infty}(\varepsilon \omega)^{k} \mathcal{N}_{p}^{ \pm}\left(U_{ \pm}^{k-m}\right)\right)=0
$$

The conditions (67) imply that the functions $c_{ \pm, p}^{2 m^{\prime}}$ are linearly independent and one deduces from that $\mathcal{N}_{p}^{ \pm}\left(U_{ \pm}^{k-2 m^{\prime}}\right)=0, \forall m^{\prime} \geqslant 0, k \geqslant 0$, that is to say

$$
\mathcal{N}_{p}^{ \pm}\left(U_{ \pm}^{k}\right)=0, \quad p \geqslant 1, \quad k \geqslant 0,
$$

which is nothing but the growth condition (37).

Next, it remains to identify power series expansions. The identification of the terms in $(\varepsilon \omega)^{k} s^{m}$, distinguishing even and odd values of $m$, leads to

$$
(\imath \omega)^{2 m^{\prime}} \mathcal{D}_{0}^{ \pm}\left(U_{ \pm}^{k}\right)=\frac{\partial^{2 m^{\prime}} u_{ \pm}^{k}}{\partial s^{2 m^{\prime}}}(0), \quad(\imath \omega)^{2 m^{\prime}+1} \mathcal{N}_{0}^{ \pm}\left(U_{ \pm}^{k}\right)=\frac{\partial^{2 m^{\prime}+1} u_{ \pm}^{k-1}}{\partial s^{2 m^{\prime}+1}}(0), \quad m^{\prime} \geqslant 0, \quad k \geqslant 0 .
$$

Using the fact that each $u_{ \pm}^{k}$ solves the 1D Helmholtz equation, we have

$$
\frac{1}{(\imath \omega)^{2 m^{\prime}}} \frac{\partial^{2 m^{\prime}} u_{ \pm}^{k}}{\partial s^{2 m^{\prime}}}=u_{ \pm}^{k}
$$

and using lemma A.3, we get the "Dirichlet" and "Neumann" matching conditions, namely (38).

\section{B. EXISTENCE AND UNIQUENESS OF THE FORMAL EXPANSION}

To prove the theorem 2.2 , we first formulate an equivalent problem where the unknowns $\left(U^{k}\right)_{k \in \mathbb{N}}$ are restricted to the junction $\widehat{J}_{\alpha}$ (section B.1). Next, we prove the existence-uniqueness result by induction on $k$ (section B.2).

\section{B.1. Restriction to a bounded domain of the problems for the $U^{k}$ 's}

Our goal in this section is to characterize the restrictions of the functions $U^{k}$ 's to the junction $\widehat{J}_{\alpha}$ by giving exact Dirichlet to Neumann boundary conditions at the interfaces $\Sigma_{ \pm}^{\alpha}$.

Using (75), (76), (78) and (81), we can write, separating $m=0$ from $m \geqslant 1$ :

$$
\begin{aligned}
U_{ \pm}^{k}=\frac{1}{\imath}\left[\int_{0}^{1} \frac{\partial U_{ \pm}^{k}}{\partial \widehat{s}}(0, \widehat{\nu}) d \widehat{\nu}\right] \mathbf{c}_{ \pm, 0}^{0} \quad+\sum_{p=0}^{\infty}\left[\int_{0}^{1} U_{ \pm}^{k}(0, \widehat{\nu}) w_{p}(\widehat{\nu}) d \widehat{\nu}\right] \mathbf{d}_{ \pm, p}^{0} \\
+\frac{1}{\imath} \sum_{m=1}^{k}\left[\int_{0}^{1} \frac{\partial U_{ \pm}^{k-m}}{\partial \widehat{s}}(0, \widehat{\nu}) d \widehat{\nu}\right] \mathbf{c}_{ \pm, 0}^{m}+\sum_{m=1}^{k} \sum_{p=0}^{\infty}\left[\int_{0}^{1} U_{ \pm}^{k-m}(0, \widehat{\nu}) w_{p}(\widehat{\nu}) d \widehat{\nu}\right] \mathbf{d}_{ \pm, p}^{m}
\end{aligned}
$$


To compute the trace of $U_{ \pm}^{k}$ and its normal derivative on $\Sigma_{ \pm}^{\alpha}$, we remark using (64) that

$$
\left\{\begin{array}{l}
\frac{\partial \mathbf{c}_{ \pm, 0}^{0}}{\partial \widehat{s}}(0, \widehat{\nu})=\imath, \quad \frac{\partial \mathbf{d}_{ \pm, 0}^{0}}{\partial \widehat{s}}(0, \widehat{\nu})=0, \\
\frac{\partial \mathbf{c}_{ \pm, 0}^{m}}{\partial \widehat{s}}(0, \widehat{\nu})=\frac{\partial \mathbf{d}_{ \pm, 0}^{m}}{\partial \widehat{s}}(0, \widehat{\nu})=0 \quad \text { for } m \geqslant 1 .
\end{array}\right.
$$

Moreover, we can deduce from (62) to (66) the existence of $\delta_{p}^{m} \in \mathbb{C}, p \geqslant 1, m \geqslant 0$ such that

$$
\frac{\partial d_{ \pm, p}^{m}}{\partial \widehat{s}}(0)= \pm \delta_{p}^{m} \quad \text { with moreover } \quad \delta_{p}^{0}=-p \pi \quad \text { and } \quad \delta_{p}^{2 m+1}=0 .
$$

and therefore that (differentiate (68) with respect to $\widehat{s}$ )

$$
\frac{\partial \mathbf{d}_{ \pm, p}^{0}}{\partial \widehat{s}}(0, \widehat{\nu})= \pm \delta_{p}^{0} w_{p}(\widehat{\nu}) \quad \text { for } p \geqslant 1, \quad k \geqslant 1 .
$$

Then, using (83) and (85), we deduce from (82) that

$$
\left\{\begin{aligned}
\frac{\partial U_{ \pm}^{k}}{\partial \widehat{s}}(0, \widehat{\nu})=\left[\int_{0}^{1} \frac{\partial U_{ \pm}^{k}}{\partial \widehat{s}}(0, \widehat{\nu}) d \widehat{\nu}\right] & -\sum_{p=1}^{\infty} p \pi\left[\int_{0}^{1} U_{ \pm}^{k}(0, \widehat{\nu}) w_{p}(\widehat{\nu}) d \widehat{\nu}\right] w_{p}(\widehat{\nu}) \\
& \pm \sum_{m=1}^{k} \sum_{p=1}^{\infty} \delta_{p}^{m}\left[\int_{0}^{1} U_{ \pm}^{k-m}(0, \widehat{\nu}) w_{p}(\widehat{\nu}) d \widehat{\nu}\right] w_{p}(\widehat{\nu})
\end{aligned}\right.
$$

Finally, using the definition of the operator $T_{ \pm}$(see (12)) and the Neumann matching condition $(38-(\mathcal{N})$ ), we see that $U^{k}$ satisfies the non homogeneous DtN condition :

$$
\frac{\partial U^{k}}{\partial n}+T_{ \pm} U^{k}= \pm \frac{1}{\omega} \frac{\partial u_{ \pm}^{k-1}}{\partial s}(0)+\sum_{m=1}^{k} \sum_{p=1}^{\infty} \delta_{p}^{m}\left[\int_{0}^{1} U_{ \pm}^{k-m}(0, \widehat{\nu}) w_{p}(\widehat{\nu}) d \widehat{\nu}\right] w_{p}(\widehat{\nu}), \quad \text { on } \Sigma_{ \pm}^{\alpha}
$$

Finally, we obtain a problem "equivalent" to $(32,33,34,35,38)$ by replacing $(38-(\mathcal{N}))$ by the DtN condition (87). The precise statement is the following

Theorem B.1. Let $\left\{\left(u_{+}^{k}, u_{-}^{k}, U^{k}\right) \in \mathrm{H}^{1}\left(\widehat{\Omega}_{+}\right) \times \mathrm{H}^{1}\left(\widehat{\Omega}_{-}\right) \times \mathrm{H}_{l o c}^{1}\left(\widehat{J}_{\alpha, \infty}\right), \quad k \geqslant 0\right\}$

be a solution of $(32,33,34,35)$ and the matching conditions (38),

$$
\left\{\left(u_{+}^{k}, u_{-}^{k}, U^{k}\right) \in \mathrm{H}^{1}\left(\widehat{\Omega}_{+}\right) \times \mathrm{H}^{1}\left(\widehat{\Omega}_{-}\right) \times \mathrm{H}^{1}\left(\widehat{J}_{\alpha}\right), \quad k \geqslant 0\right\}
$$

is solution of $(32,33),(34,35)_{\widehat{J}_{\alpha}},(87)$ and $(38-(\mathcal{D}))$

where $(34,35)_{\widehat{J}_{\alpha}}$ holds for the restrictions of equations $(34,35)$ respectively to $\widehat{J}_{\alpha}$ and $\partial \widehat{J}_{\alpha, \infty} \cap \partial \widehat{J}_{\alpha}$.

Reciprocally, if $\left\{\left(u_{+}^{k}, u_{-}^{k}, U^{k}\right) \in \mathrm{H}^{1}\left(\widehat{\Omega}_{+}\right) \times \mathrm{H}^{1}\left(\widehat{\Omega}_{-}\right) \times \mathrm{H}^{1}\left(\widehat{J}_{\alpha}\right), \quad k \geqslant 0\right\}$

is solution of $(32,33),(34,35)_{\widehat{J}_{\alpha}},(87)$ and $(38-(\mathcal{D}))$, then, extending $U^{k}$ to $\widehat{B}_{ \pm}$via (82),

$$
\left\{\left(u_{+}^{k}, u_{-}^{k}, U^{k}\right) \in \mathrm{H}^{1}\left(\widehat{\Omega}_{+}\right) \times \mathrm{H}^{1}\left(\widehat{\Omega}_{-}\right) \times \mathrm{H}_{l o c}^{1}\left(\widehat{J}_{\alpha, \infty}\right), \quad k \geqslant 0\right\}
$$

is a solution of $(32,33,34,35)$ and (38). 
Proof. The direct statement has been proven. The proof of the converse statement is rather tedious but not classical. See [2] for details.

\section{B.2. Existence and Uniqueness}

It is done by induction on $k$. According to theorem B.1, it suffices to consider the problem $(32,33),(34,35) \widehat{J}_{\alpha}$, $(87)$ and $(38-(\mathcal{D}))$. To clearly identify the recursion process, it is useful to reformulate this problem in a more decoupled way (we mean between $u_{ \pm}^{k}$ and $U^{k}$, at each step $k$ ), which is also useful from the computational point of view.

To achieve such a decoupling the idea is first to consider (87) as a boundary condition for $U_{ \pm}^{k}$, next to formulate a 1D transmission problem for $u_{ \pm}^{k}$. That is why we shall use the following two technical lemmas

Lemma B.2. Given $\Phi \in \mathrm{L}^{2}\left(\widehat{J}_{\alpha}\right)$ and $g_{ \pm} \in \mathrm{H}^{-\frac{1}{2}}\left(\Sigma_{ \pm}^{\alpha}\right)$, there exists $U \in \mathrm{H}^{1}\left(\widehat{J}_{\alpha}\right)$, which is unique up to an additive constant, such that there exists $U \in \mathrm{H}^{1}\left(\widehat{J}_{\alpha}\right)$, which is unique up to an additive constant, such that

$$
\begin{cases}\Delta U=\Phi, & \text { in } \widehat{J}_{\alpha}, \quad \frac{\partial U}{\partial n}=0, \quad \text { on } \partial \widehat{J}_{\alpha} \backslash\left(\Sigma_{+}^{\alpha} \cap \Sigma_{-}^{\alpha}\right) . \\ \frac{\partial U}{\partial n}+T_{ \pm} U=g_{ \pm}, & \text {on } \Sigma_{ \pm}^{\alpha},\end{cases}
$$

if and only if one satisfies the compatibility condition

$$
\int_{\Sigma_{-}^{\alpha}} g_{-}+\int_{\Sigma_{+}^{\alpha}} g_{+}=\int_{\widehat{J}_{\alpha}} \Phi
$$

Moreover, $\mathcal{W}_{\alpha}$ being defined by $(11,10)$, any solution of (88) satisfies

$$
\int_{\Sigma_{+}^{\alpha}} U-\int_{\Sigma_{-}^{\alpha}} U=\int_{\widehat{J}_{\alpha}} \Phi \mathcal{W}_{\alpha}+\int_{\Sigma_{-}^{\alpha}} g_{-} \mathcal{W}_{\alpha}+\int_{\Sigma_{+}^{\alpha}} g_{+} \mathcal{W}_{\alpha} .
$$

Proof. The existence-uniqueness proof is a classical exercise about Lax-Milgram's lemma and Poincaré-Wirtinger's inequality (the important point is that $T_{ \pm}: \mathrm{H}^{\frac{1}{2}}\left(\Sigma_{ \pm}^{\alpha}\right) \mapsto \mathrm{H}^{-\frac{1}{2}}\left(\Sigma_{ \pm}^{\alpha}\right)$ is a positive symmetric operator whose kernel is the space of constant functions). The compatibility condition (89) is obtained by integrating the first equation of (88), using Green's formula and the symmetry of $T_{ \pm}$.

To obtain (90), we multiply the equation for $U$ by $\mathcal{W}_{\alpha}$ and integrate over $\widehat{J}_{\alpha}$. Using Green's formula twice, and the fact that $\mathcal{W}_{\alpha}$ is harmonic, we get

$$
\sum_{ \pm} \int_{\Sigma_{ \pm}^{\alpha}}\left(\frac{\partial \mathcal{W}_{\alpha}}{\partial n} U-\frac{\partial U}{\partial n} \mathcal{W}_{\alpha}\right)=\int_{\widehat{J}_{\alpha}} \Phi \mathcal{W}_{\alpha}
$$

Using the boundary conditions on $\Sigma_{ \pm}^{\alpha}$ for $U$ and $\mathcal{W}_{\alpha}$ together with the symmetry of $T_{ \pm}$, we obtain (89).

Lemma B.3. Given $\left(j_{d}, j_{n}\right) \in \mathbb{C} \times \mathbb{C}$ and $f_{-} \in \mathbb{C}$, there exists a unique $u_{ \pm} \in \mathrm{H}^{1}\left(\left(0, \pm L_{ \pm}\right)\right)$, such that:

$$
\begin{cases}\frac{\partial^{2} u_{ \pm}}{\partial s^{2}}+\omega^{2} u_{ \pm}=0, & \pm s \in\left[0, L^{ \pm}[,\right. \\ \left(\frac{\partial u_{+}}{\partial s}+\imath \omega u_{+}\right)\left(L_{+}\right)=0, & \frac{\partial u_{-}}{\partial s}\left(L_{-}\right)=f_{-}, \\ u_{+}(0)-u_{-}(0)=j_{d}, & \frac{\partial u_{+}}{\partial s}(0)-\frac{\partial u_{-}}{\partial s}(0)=j_{n} .\end{cases}
$$


Proof. The result is straightforward.

According to $(34,35)_{\widehat{J}_{\alpha}}$ and $(87)$, we can apply lemma B.2 with $U=U^{k}, \Phi=-U^{k-2}$ and $g_{ \pm}=g_{ \pm}^{k-1}$ with

$$
g_{ \pm}^{k-1}:= \pm \frac{1}{\omega} \frac{\partial u_{ \pm}^{k-1}}{\partial s}(0)+\sum_{m=1}^{k} \sum_{p=1}^{\infty} \delta_{p}^{m}\left[\int_{0}^{1} U_{ \pm}^{k-m}(0, \widehat{\nu}) w_{p}(\widehat{\nu}) d \widehat{\nu}\right] w_{p}(\widehat{\nu}), \quad \text { on } \Sigma_{ \pm}^{\alpha}
$$

where the index $k-1$ in $g_{ \pm}^{k-1}$ is "justified" by the fact that $g_{ \pm}^{k-1}$ is known explicitly when $u_{ \pm}^{k-1}$ and the $U^{m}$ 's for $m \leqslant k-1$ are known. Writing (89) gives

$$
\frac{1}{\omega}\left(\frac{\partial u_{ \pm}^{k-1}}{\partial s}\left(0^{+}\right)-\frac{\partial u_{ \pm}^{k-1}}{\partial s}\left(0^{-}\right)\right)=-\int_{\widehat{J}_{\alpha}} U^{k-2} .
$$

which, written for " $k=k+1$ ", gives the Neumann jump condition

$$
\frac{1}{\omega}\left(\frac{\partial u_{ \pm}^{k}}{\partial s}\left(0^{+}\right)-\frac{\partial u_{ \pm}^{k}}{\partial s}\left(0^{-}\right)\right)=-\int_{\widehat{J}_{\alpha}} U^{k-1}, \quad k \geqslant 0 .
$$

Writing (90) gives the Dirichlet jump condition

$$
\int_{\Sigma_{+}^{\alpha}} U^{k}-\int_{\Sigma_{-}^{\alpha}} U^{k}=-\int_{\widehat{J}_{\alpha}} U^{k-2} \mathcal{W}_{\alpha}+\int_{\Sigma_{-}^{\alpha}} g_{-}^{k-1} \mathcal{W}_{\alpha}+\int_{\Sigma_{+}^{\alpha}} g_{+}^{k-1} \mathcal{W}_{\alpha} .
$$

that can be rewritten, using the matching conditions $(38-(\mathcal{D}))$ :

$$
u_{ \pm}^{k}\left(0^{+}\right)-u_{ \pm}^{k}\left(0^{-}\right)=-\int_{\widehat{J}_{\alpha}} U^{k-2} \mathcal{W}_{\alpha}+\int_{\Sigma_{-}^{\alpha}} g_{-}^{k-1} \mathcal{W}_{\alpha}+\int_{\Sigma_{+}^{\alpha}} g_{+}^{k-1} \mathcal{W}_{\alpha} .
$$

For each $k$, we have succeeded to decouple the calculation of $u_{ \pm}^{k}$ since jump conditions (93) and (95) written for " $k=k+1$ " are sufficient, when associated to equations $(32,33)$, to determine $u_{ \pm}^{k}$ uniquely (lemma B.3).

As the solution of problem (P) with $\Phi=U^{k-2}$ and $g_{ \pm}=g_{ \pm}^{k-1}, U^{k}$ is defined up to an additive constant. To fix this constant we can use again $(38-(\mathcal{D})$ ) (in a symmetric way with respect to \pm ) :

$$
\frac{1}{2}\left(\int_{\Sigma_{+}^{\alpha}} U^{k}+\int_{\Sigma_{-}^{\alpha}} U^{k}\right)=\frac{1}{2}\left(u_{+}^{k}\left(0^{+}\right)+u_{-}^{k}\left(0^{-}\right)\right)
$$

Finally, we obtain an equivalent problem to $(32,33),(34,35)_{\widehat{J}_{\alpha}},(87),(38-(\mathcal{D}))$ by replacing $(38-(\mathcal{D}))$ by $(93)$, (95) and (96). More precisely

Theorem B.4. The following two propositions are equivalent (for the clarity of notation, we omit to mention again the functional setting):

(i) $\left\{\left(u_{+}^{k}, u_{-}^{k}, U^{k}\right), k \geqslant 0\right\}$ is solution of $(32,33),(34,35)_{\widehat{J}_{\alpha}},(87)$ and $(38-(\mathcal{D}))$.

(ii) $\left\{\left(u_{+}^{k}, u_{-}^{k}, U^{k}\right), k \geqslant 0\right\}$ is solution of $(32,33,93,95)$ and $\left((34,35)_{\widehat{J}_{\alpha}}, 87,96\right)$, with $g_{ \pm}^{k-1}$ defined as (92).

Proof. We just proved the implication $(i) \Longrightarrow(i i)$. The proof of $(i i) \Longrightarrow(i)$ is similar (see [2] for more details). 
The case $k=0$. With the convention of remark 2.1 , we see from $(32,33,93,95)$ that $u_{ \pm}^{0}$ is, as expected, the solution of the transmission problem for the 1D Helmholtz problem with the transmission conditions (9) with $\left.u^{0}\left(0^{ \pm}\right) \equiv u_{ \pm}^{0}(0)\right)$, i. e. the "concatenation" of $u_{-}^{0}$ and $u_{+}^{0}$ is nothing but $u^{0}$ as defined in section 1.2 (see (4)).

Moreover, we see from $(34,35)$ and $(87)$ that $U^{0}$ solves $(88)$ with $\Phi=0$ and $g_{ \pm}=0$, which implies that $U^{0}$ is constant. Next, (96) gives

$$
U^{0}=\frac{1}{2}\left(u_{+}^{0}(0)+u_{-}^{0}(0)\right)=u^{0}(0)
$$

The general case $k \geqslant 1$. Assume that $\left(u_{+}^{\ell}, u_{-}^{\ell}, U^{\ell}\right), \ell \leqslant k-1$ are known, then, according to theorem B.4,

- We first determine $\left(u_{+}^{k}, u_{-}^{k}\right)$ as the unique solution of the $1 \mathrm{D}$ transmission problem $(32,33)$ with the transmission conditions $(93,95)$. (cf. lemma B.3)

- We compute $g_{ \pm}^{k-1}$ thanks to $(92)$.

- We determine $U^{k}$ as the solution, cf. lemma B.2, of the boundary value problem $\left((34,35)_{\widehat{J}_{\alpha}}, 87,96\right)$. One must of course check the compatibility condition (89), which is a consequence of (93).

Finally, regrouping the above results with theorems B.1 and B.4, we have proven the theorem 2.2.

\section{REFERENCES}

[1] Arlen M. Il'in. Matching of asymptotic expansions of solutions of boundary value problems, volume 102 of Translations of Mathematical Monographs. American Mathematical Society, Providence, RI, 1992. Translated from the Russian by V. Minachin [V. V. Minakhin].

[2] Patrick Joly and Adrien Semin. Propagation of an acoustic wave in a junction of two thin slots. INRIA Research Report, pages R1-R61, 2008 (submitted).

[3] Patrick Joly and Sébastien Tordeux. Matching of asymptotic expansions for wave propagation in media with thin slots. I. The asymptotic expansion. Multiscale Model. Simul., 5(1):304-336 (electronic), 2006.

[4] Joseph B. Keller and Dan Givoli. Exact nonreflecting boundary conditions. J. Comput. Phys., 82(1):172-192, 1989.

[5] Peter Kuchment. Graph models for waves in thin structures. Waves Random Media, 12(4):R1-R24, 2002.

[6] Paul A. Martin and Robert A. Dalrymple. Scattering of long waves by cylindrical obstacles and gratings using matched asymptotic expansions. J. Fluid Mech., 188:465-490, 1988.

[7] Vladimir Maz'ya, Serguei Nazarov, and Boris Plamenevskij. Asymptotic theory of elliptic boundary value problems in singularly perturbed domains. Vol. I, volume 111 of Operator Theory: Advances and Applications. Birkhäuser Verlag, Basel, 2000. Translated from the German by Georg Heinig and Christian Posthoff.

[8] Jacob Rubinstein and Michelle Schatzman. Variational problems on multiply connected thin strips. I. Basic estimates and convergence of the Laplacian spectrum. Arch. Ration. Mech. Anal., 160(4):271-308, 2001.

[9] Jacob Rubinstein and Michelle Schatzman. Variational problems on multiply connected thin strips. II. Convergence of the Ginzburg-Landau functional. Arch. Ration. Mech. Anal., 160(4):309-324, 2001.

[10] Sébastien Tordeux, Grégory Vial, and Monique Dauge. Matching and multiscale expansions for a model singular perturbation problem. C. R. Math. Acad. Sci. Paris, 343(10):637-642, 2006.

[11] Milton Van Dyke. Perturbation methods in fluid mechanics. Applied Mathematics and Mechanics, Vol. 8. Academic Press, New York, 1964. 